\title{
STRATIFICATIONS OF FLAG SPACES AND FUNCTORIALITY
}

\author{
WUSHI GOLDRING, JEAN-STEFAN KOSKIVIRTA
}

\begin{abstract}
We define stacks of zip flags, which form towers above the stack of $G$-zips of Moonen, Pink, Wedhorn and Ziegler in MW04, PWZ11 and PWZ15. A stratification is defined on the stack of zip flags, and principal purity is established under a mild assumption on the underlying prime $p$. We generalize flag spaces of Ekedahl-Van der Geer EvdG09 and relate them to stacks of zip flags. For large $p$, it is shown that strata are affine. We prove that morphisms with central kernel between stacks of G-zips have discrete fibers. This allows us to prove principal purity of the zip stratification for maximal zip data. The latter provides a new proof of the existence of Hasse invariants for Ekedahl-Oort strata of good reduction Shimura varieties of Hodge-type, first proved in GKb.
\end{abstract}

\section{INTRODUCTION}

This paper is the second in a series on our program, outlined in [GKb, to connect (A) Automorphic Algebraicity, (B) G-Zip Geometricity and (C) Griffiths-Schmid Algebraicity. The two main themes motivating our program are those of geometry-by-groups and characteristic shifting. For more details on our program and background on (A), (B), (C), see the introduction of loc. cit. and the references therein.

The specific focus of this paper is to dig deeper into several aspects of (B) which were studied in loc. cit. More precisely, four interrelated topics are pursued:

(1) The behavior of the zip stratification under functoriality.

(2) The construction of stacks $G$-ZipFlag ${ }^{\left(\mathcal{Z}, P_{0}\right)}$ of zip partial flags, together with a stratification of EkedahlOort type. The stacks $G$-ZipFlag ${ }^{\left(\mathcal{Z}, P_{0}\right)}$ lie between a stack of $G$-Zips and the stack of zip flags $G$-ZipFlag ${ }^{\mathcal{Z}}$ defined in $\mathrm{GKb}$ :

$$
G \text {-ZipFlag }{ }^{\mathcal{Z}} \rightarrow G \text {-ZipFlag }{ }^{\left(\mathcal{Z}, P_{0}\right)} \rightarrow G \text {-Zip }{ }^{\mathcal{Z}}
$$

(3) The construction of group-theoretical Hasse invariants on the above stacks, under mild bounds on the characteristic. We view the existence of such Hasse invariants in terms of several strengthened notions of purity.

(4) The construction of partial flag spaces, which lie in-between a Shimura variety of Hodge type and its flag space.

The main results obtained in each of the four areas singled out above are summarized below. One of the technical innovations in this paper over [GKb is the use of zip data of higher exponent $n$ (corresponding to the $n$th power of Frobenius, see 1.1), while loc. cit. only considered zip data of exponent 1. Passing to a higher exponent is a key method in Th. 2 below.

Another improvement is that a criterion is given for the existence of group-theoretical Hasse invariants on a general zip datum, while loc. cit. only treated those zip data arising from cocharacters. This generalization is necessary to obtain sections on the strata of $G$-ZipFlag ${ }^{\left(\mathcal{Z}, P_{0}\right)}$.

The above topics have already had numerous applications. Some applications to the coherent cohomology of Shimura varieties, automorphic forms and the Langlands Program were given in [GKb]. Applications to the existence of global sections of automorphic vector bundles, in particular proving a conjecture of Diamond on mod $p$ Hilbert modular forms were given in GKa. The second author has applied the theory of partial flag spaces developed here to determine the normalization of Ekedahl-Oort strata closures [Kos]. For additional applications to ampleness of automorphic bundles and vanishing theorems for the coherent cohomology of Shimura varieties, see forthcoming work with Brunebarbe and Stroh [BGKS]. We expect the above applications to be merely the tip of the iceberg: many more will follow.

Stacks of zip flags and their stratification. Let $G$ be a connected, reductive $\mathbf{F}_{p}$-group; let $\mathcal{Z}=\left(G, P, L, Q, M, \varphi^{n}\right)$ be a zip datum and let $G$-Zip ${ }^{\mathcal{Z}}$ be the associated stack of $G$-Zips (\$1.1). The following summarizes some of the basic constructions in $\$ 2$ and $\$ 3.1$

Theorem 1. Let $P_{0}$ be a sub-parabolic of $P$.

(1) There exists a smooth stack $G$-ZipFlag ${ }^{\left(\mathcal{Z}, P_{0}\right)}$ of dimension $\operatorname{dim}\left(P / P_{0}\right)$ of zip flags of type $P_{0}$.

(2) There is a natural projection $G$-ZipFlag ${ }^{\left(\mathcal{Z}, P_{0}\right)} \rightarrow G$-Zip ${ }^{\mathcal{Z}}$, which is smooth with flag variety fibers $P / P_{0}$. 
(3) If $B \subset P_{0}$ is a Borel subgroup, then the projection $G$-ZipFlag $\mathcal{Z}^{\mathcal{Z}} \rightarrow G$-Zip ${ }^{\mathcal{Z}}$ factors through $G$-ZipFlag ${ }^{\mathcal{Z}} \rightarrow$ $G$-ZipFlag ${ }^{\left(\mathcal{Z}, P_{0}\right)}$, with flag variety fibers $P_{0} / B$.

(4) There is a second zip datum $\mathcal{Z}_{0}$ associated to $\left(\mathcal{Z}, P_{0}\right)$ and a smooth map $G$-ZipFlag ${ }^{\left(\mathcal{Z}, P_{0}\right)} \rightarrow G$-Zip ${ }^{\mathcal{Z}_{0}}$. It induces a stratification of $G$-ZipFlag ${ }^{\left(\mathcal{Z}, P_{0}\right)}$ parameterized by ${ }^{I_{0}} W$, where $I_{0}$ is the type of $P_{0}$.

The stratification in (4) is termed fine, see $\$ 3.1$. We also define a coarse stratification of $G$-ZipFlag ${ }^{\left(\mathcal{Z}, P_{0}\right)}$, see \$2.2, it generalizes the Bruhat stratification of $G$-Zip ${ }^{\mathcal{Z}}$ studied by Wedhorn Wed14, which in turn generalizes the a-number stratification studied by Oort and others (cf. introduction of loc. cit.).

In general, the zip datum $\mathcal{Z}_{0}$ is not attached to a cocharacter, unless $P_{0}=P$. There seems to be no known algebraic counterpart of the stack $G$-Zip ${ }^{\mathcal{Z}_{0}}$ in the theory of Shimura varieties. Thus we see that the group-theoretic approach of $G$-Zips reveals genuinely new structure. Nevertheless, following (i) the analogy between stacks of $G$ Zips and Griffiths-Schmid manifolds suggested in GKb] (see esp. §I.4) and (ii) the approach to Griffiths-Schmid manifolds in [GGK13, it appears that there may be a complex-geometric analogue of $G$-Zip ${ }^{\mathcal{Z}_{0}}$ among the objects studied in loc. cit.; see 6.4 .

Th. 1 generalizes the construction of the stack of zip flags $G$-ZipFlag ${ }^{\mathcal{Z}}$ in GKb to an intermediate parabolic $B \subset P_{0} \subset P$. In particular, when $P_{0}=B$ one has $G$-ZipFlag ${ }^{\left(\mathcal{Z}, P_{0}\right)}=G$-ZipFlag ${ }^{\mathcal{Z}}$ and when $P_{0}=P$ one has $G$-ZipFlag ${ }^{\left(\mathcal{Z}, P_{0}\right)}=G$-Zip ${ }^{\mathcal{Z}}$. In turn, the case of $G$-ZipFlag ${ }^{\mathcal{Z}}$ is a group-theoretical generalization of the flag space - and its stratification - associated to the moduli space of abelian varieties $\mathcal{A}_{g} \otimes \mathbf{F}_{p}$ by Ekedahl and van der Geer [EvdG09].

Functoriality of zip stratifications. The association $\mathcal{Z} \rightarrow G$-Zip ${ }^{\mathcal{Z}}$ is functorial: A morphism $f: \mathcal{Z}_{1} \rightarrow \mathcal{Z}_{2}$ of zip data is a morphism of the underlying groups $f: G_{1} \rightarrow G_{2}$, compatible with the additional structure (see $\$ 1.1$ ). Such a morphims induces one of stacks $\tilde{f}: G_{1}$-Zip $^{\mathcal{Z}_{1}} \rightarrow G_{2}$-Zip $^{\mathcal{Z}_{2}}$ (Lemma 1.5.3). It is then natural to question how the zip stratification varies under functoriality.

Theorem 2 (Th. 5.1.1). Let $f: \mathcal{Z}_{1} \rightarrow \mathcal{Z}_{2}$ be a morphism of zip data, such that the underlying morphism of groups $f: G_{1} \rightarrow G_{2}$ has central kernel. Then the induced morphism of stacks

$$
\tilde{f}: G_{1} \text { Zip }^{\mathcal{Z}_{1}} \rightarrow G_{2} \text {-Zip }^{\mathcal{Z}_{2}}
$$

has discrete fibers on the underlying topological spaces.

Concretely, the discreteness of fibers means that, if two strata $S$ and $S^{\prime}$ of $G_{1}$-Zip ${ }^{\mathcal{Z}_{1}}$ map into the same stratum of $G_{2}$-Zip ${ }^{\mathcal{Z}_{2}}$, then $S$ and $S^{\prime}$ are incomparable in the zip stratification of $G_{1}$-Zip ${ }^{\mathcal{Z}_{1}}$ (neither is contained in the closure of the other).

An immediate application of Th. 2 is that the same type of functoriality is satisfied by the Ekedahl-Oort stratification of Hodge-type Shimura varieties.

(Uniform) principal purity of strata. Recall that a stratification on a scheme (or stack) $X$ is principally pure if each stratum $S \subset X$ is the non-vanishing locus of a section of a line bundle on the Zariski closure $\bar{S}$. We call such a section a characteristic section for $S$. In case $S$ is a stratum of $G$-ZipFlag ${ }^{\left(\mathcal{Z}, P_{0}\right)}$, due to the connection with the classical Hasse invariant of an abelian scheme, we also call such a section a group-theoretical Hasse invariant.

The following question is central to this work:

Question. For which zip data $\mathcal{Z}$ is the zip stratification of $G$-Zip ${ }^{\mathcal{Z}}$ principally pure? More generally, for which $\left(\mathcal{Z}, P_{0}\right)$ is the fine stratification of $G$-ZipFlag ${ }^{\left(\mathcal{Z}, P_{0}\right)}$ principally pure?

An example where the zip stratification is not principally pure is given in 4.3 . However, the following shows that such examples are extremely rare. In fact, the result below shows that a stronger property than principal purity usually holds. We say that a stratification of a scheme (or stack) is uniformly principally pure if there exists a line bundle that admits characteristic sections for all strata.

Let $\left(\mathcal{Z}, P_{0}\right)$ be a pair as in Th. 1, with associated zip datum $\mathcal{Z}_{0}$. For a character $\lambda \in X^{*}\left(P_{0}\right)$, we define properties "orbitally $q$-close" (Def. 4.1.2) and " $\mathcal{Z}_{0}$-ample" (Def. 4.1.3). Then one has:

Theorem 3 (Th. 4.2.4). Let $q=p^{n}$. If there exists a $\mathcal{Z}_{0}$-ample, orbitally $q$-close character of $P_{0}$, then the fine stratification of $G$-ZipFlag ${ }^{\left(\mathcal{Z}, P_{0}\right)}$ is uniformly principally pure.

Th. 2 and Th. [3 are closely related. To prove the former, we use the latter as a key ingredient. Conversely, Th. 2 has consequences for the existence of characteristic sections for maximal zip data (see below and \$1.4), and in particular for Hodge-type zip data (Rmk. 1.4.4).

We say that a cocharacter datum $(G, \mu)$ is maximal if there exists a representation $r: G \rightarrow G L(V)$ with central kernel such that $\mu \circ r$ has exactly two weights. This implies that the parabolic subgroup of $G L(V)$ attached to $r \circ \mu$ is maximal. A zip datum $\mathcal{Z}$ is called maximal if $\mathcal{Z}=\mathcal{Z}_{\mu}$ for some maximal $(G, \mu)$. For example, a Shimura datum with good reduction at $p$ gives rise to a maximal cocharacter datum. We prove the following: 
Corollary 1 (Cor. 5.2.2). If $(G, \mu)$ is maximal, then the stratification of $G$-Zip ${ }^{\mu}$ is uniformly principally pure.

This gives another proof of the existence of Hasse invariants for good reduction Shimura varieties of Hodge-type, first proved in GKb. In loc. cit. Cor. 3.1.3, we proved the special case of Cor. 1, when $(G, \mu)$ arises by reduction modulo $p$ from a Shimura datum of Hodge-type. The proof in loc. cit. used the methods of [Del79] to show that the Hodge line bundle was orbitally $p$-close. By contrast with [GKb], no characteristic zero methods are used here and no case-by-case examination is needed.

Flag spaces and affineness of strata. In [GKb, we generalized the construction of the flag space of EvdG09] to arbitrary Hodge-type Shimura varieties. Here we generalize this construction further to arbitrary parabolics.

Let $(\mathbf{G}, X)$ be a Shimura datum of Hodge-type. Let $\mathscr{S}_{K}$ be the Kisin-Vasiu integral model of the associated Shimura variety $S h_{K}(\mathbf{G}, X)$ at a level $K$ which is hyperspecial at $p$. Denote the special fiber of $\mathscr{S}_{K}$ by $S_{K}$. Recall that Zhang Zha] gives a smooth morphism $\zeta: S_{K} \rightarrow G$-Zip ${ }^{\mathcal{Z}}$, where $G$ is the special fiber of an extension of $\mathbf{G}$ to a smooth, reductive group scheme over $\mathbf{Z}_{(p)}$.

Associated to a sub-parabolic $P_{0} \subset P$, we define a partial flag space $\mathcal{F} l_{K}$. It is a fibration over $\mathscr{S}_{K}$ with fiber $P / P_{0}$. The special fiber $F l_{K}$ of $\mathcal{F} l_{K}$ fits in a cartesian diagram:

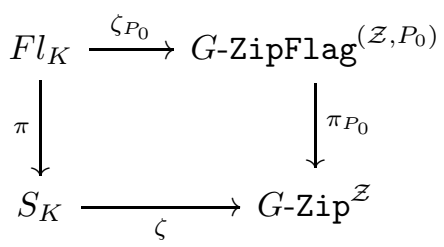

Fine flag strata of $F l_{K}$ are defined as the preimages by $\zeta_{P_{0}}$ of those in $G$-ZipFlag ${ }^{\left(\mathcal{Z}, P_{0}\right)}$.

Theorem 4 (Th. 6.3.2). There exists an integer $N=N(\mathbf{G}, X, K)$ such that, for all $p \geq N$, one has:

(1) $S h_{K}(\mathbf{G}, X)$ has good reduction at all places $\mathfrak{p} \mid p$.

(2) The fine stratification of $F l_{K}$ is uniformly principally pure.

(3) If the closure $\bar{S}$ of a fine flag stratum $S \subset F l_{K}$ is proper, then $S$ is affine.

The special case where the Shimura variety is of Siegel-type and $P_{0}=B$ was treated in [EvdG09, Prop.10.5 ii)]. Note that the hypothesis of (2) is satisfied if $S_{K}$ is proper.

Outline. $\S 1$ reviews the theory of $G$-zips, cocharacter data and zip data, and explains the relation between them. Maximal cocharacter data are also defined. Finally, we recall basic facts on the stack $G$-Zip ${ }^{\mathcal{Z}}$, and give a parametrization of the strata.

In $\S 2$, we introduce the stack of $G$-zip flags, represent it as a quotient stack and define coarse flag strata. $\S 3$ is devoted to the definition of fine flag strata. The important notions of minimal and cominimal strata are defined. $\S 4$ studies sections of line bundles on fine flag strata and zip strata. In particular, Th. 4.2.4 is proved and a counterexample to principal purity is given. Some aspects of functoriality are investigated in $\S 5$. The main results are Th. 5.1.1 on discreteness of fibers and Cor. 5.2.2 for maximal zip data.

We look at some applications to Shimura varieties in $\S 6$, where generalized flag spaces and their stratifications are defined. Finally, Th. 6.3.2 is proved.

\section{ACKNowledgments}

The authors would like to thank Torsten Wedhorn for very stimulating discussions and advice. We are grateful to Benoît Stroh for important ideas that influenced some aspects of the paper. Also, we thank Paul Ziegler for helpful discussions and correspondence. Finally we want to thank the reviewers for their suggestions on how to improve the paper.

\section{REview OF G-ZIPS}

We fix a prime number $p$ and denote by $k$ an algebraic closure of $\mathbf{F}_{p}$.

1.1. The category of zip data. Let $n \geq 1$ be an integer. In this paper, a zip datum of exponent $n$ is a tuple $\mathcal{Z}=\left(G, P, L, Q, M, \varphi^{n}\right)$, where $G$ is a reductive group over $\mathbf{F}_{p}, \varphi: G \rightarrow G$ the relative Frobenius morphism, $P, Q \subset G_{k}$ parabolics (not necessarily defined over $\mathbf{F}_{p}$ ) with Levi subgroups $L \subset P$ and $M \subset Q$, and $n \geq 1$ an integer. We impose the condition $M=\varphi^{n}(L)$. Hence the $p^{n}$-Frobenius map restricts to $\varphi^{n}: L \rightarrow M$.

Zip data of exponent $n$ form a category $\mathscr{D}_{n}$, where morphisms are defined as follows. Let $\mathcal{Z}_{i}=\left(G, P_{i}, L_{i}, Q_{i}, M_{i}, \varphi^{n}\right)$ for $i=1,2$ two zip data of exponent $n$, and denote by $U_{i} \subset P_{i}$ and $V_{i} \subset Q_{i}$ the unipotent radicals. A morphism of zip data $\mathcal{Z}_{1} \rightarrow \mathcal{Z}_{2}$ is a group homomorphism $f: G_{1} \rightarrow G_{2}$ (defined over $\mathbf{F}_{p}$ ) satisfying the conditions

$$
f\left(\square_{1}\right) \subset \square_{2} \text { for } \square=P, L, Q, M, U, V .
$$


For a zip datum $\mathcal{Z}=\left(G, P, L, Q, M, \varphi^{n}\right)$ and $g \in G(k)$, we define a conjugate zip datum

$$
{ }^{g} \mathcal{Z}:=\left(G,{ }^{g} P,{ }^{g} L, \varphi^{n}(g) Q, \varphi^{n}(g) M, \varphi^{n}\right) .
$$

1.2. Cocharacter data. Let $K$ be a field with a fixed algebraic closure $\bar{K}$. We define the category of cocharacter data over a field $K$ as the category of pairs $(G, \mu)$ where $G$ is a reductive group over $K$ and $\mu: \mathbf{G}_{m, \bar{K}} \rightarrow G_{\bar{K}}$ is a cocharacter.

The category of cocharacter data is denoted by $\mathscr{D}^{\mathrm{co}}$. Morphisms of cocharacter data $\left(G_{1}, \mu_{1}\right) \rightarrow\left(G_{2}, \mu_{2}\right)$ are group homomorphisms $f: G_{1} \rightarrow G_{2}$ (defined over $K$ ) satisfying $\mu_{2}=f \circ \mu_{1}$.

Let $(G, \mu)$ be a cocharacter datum. The cocharacter $\mu$ gives rise to a pair of opposite parabolics $\left(P_{-}, P_{+}\right)$and a Levi subgroup $L:=P_{-} \cap P_{+}$. The Lie algebra of the parabolic $P_{-}$(resp. $P_{+}$) is the sum of the non-positive (resp. non-negative) weight spaces of Ad $\circ \mu$. More precisely, $P_{+}(\bar{K})$ consists of elements $g \in G(\bar{K})$ such that the limit

$$
\lim _{t \rightarrow 0} \mu(t) g \mu(t)^{-1}
$$

exists, i.e such that the map $\mathbf{G}_{m, \bar{K}} \rightarrow G_{\bar{K}}, t \mapsto \mu(t) g \mu(t)^{-1}$ extends to a morphism of varieties $\mathbf{A}_{\bar{K}} \rightarrow G_{\bar{K}}$. The unipotent radical of $P_{+}$is the set of such elements $g$ for which this limit is $1 \in G(\bar{K})$.

When $K=\mathbf{F}_{p}$, we define $P:=P_{-}, Q:=\varphi^{n}\left(P_{+}\right)$and $M:=\varphi^{n}(L)$. The tuple $\mathcal{Z}_{\mu}:=\left(G, P, L, Q, M, \varphi^{n}\right)$ is then a zip datum of exponent $n$. We call it the zip datum of exponent $n$ attached to the cocharacter datum $(G, \mu)$.

Proposition 1.2.1. The construction above gives rise to a faithful functor

$$
\mathcal{Z}_{n}: \mathscr{D}^{\mathrm{co}} \longrightarrow \mathscr{D}_{n},(G, \mu) \mapsto \mathcal{Z}_{\mu}
$$

Proof. This follows immediately from characterization (1.2.1) above.

1.3. Frames of zip data. Let $\mathcal{Z}:=\left(G, P, L, Q, M, \varphi^{n}\right)$ be a zip datum of exponent $n \geq 1$. Let $(B, T)$ be a Borel pair in $G$ defined over $\mathbf{F}_{p}$, which exists by Steinberg's Theorem.

Write $W:=W(G, T)$ for the Weyl group of $G_{k}$. Let $\Phi \subset X^{*}(T)$ (resp. $\Phi_{L} \subset X^{*}(T)$ ) be the set of $T$-roots of $G$ (resp. $L$ ). We define the set of positive roots $\Phi^{+} \subset \Phi$ by the condition that $\alpha \in \Phi^{+}$when $U_{\alpha} \subset B$. Write $\Delta \subset \Phi^{+}$ for the set of positive simple roots. For $\alpha \in \Phi$, let $s_{\alpha} \in W$ be the corresponding reflection. Then $\left(W,\left\{s_{\alpha}, \alpha \in \Delta\right\}\right)$ is a Coxeter group and we denote by $\ell: W \rightarrow \mathbf{N}$ the length function.

Let $I \subset \Delta$ (resp. $J \subset \Delta$ ) be the type of $P$ (resp. $Q$ ). For any subset $K \subset \Delta$, let $W_{K} \subset W$ be the subgroup generated by $\left\{s_{\alpha}, \alpha \in K\right\}$. Let $w_{0}$ (resp. $w_{0, K}$ ) be the longest element in $W$ (resp. $W_{K}$ ). Denote by ${ }^{K} W$ (resp. $\left.W^{K}\right)$ the subset of elements $w \in W$ which are minimal in the coset $W_{K} w$ (resp. $\left.w W_{K}\right)$. The set ${ }^{K} W\left(\right.$ resp. $\left.W^{K}\right)$ is a set of representatives for the quotient $W_{K} \backslash W\left(\operatorname{resp} . W / W_{K}\right)$.

Definition 1.3.1. Let $(B, T)$ be a Borel pair and $z \in W$. We call $(B, T, z)$ a $W$-frame for $\mathcal{Z}$ if the following conditions are satisfied:

(i) B,T are defined over $\mathbf{F}_{p}$.

(ii) $B \subset P$.

(iii) ${ }^{z} B \subset Q$.

(iv) $\varphi(B \cap L)={ }^{z} B \cap M$.

Remark 1.3.2.

(1) If $(B, T)$ is defined over $\mathbf{F}_{p}$, there exists $z \in W$ such that $(B, T, z)$ is a $W$-frame (proof of [PWZ11, Prop. 3.7]).

(2) For any zip datum $\mathcal{Z}$, there exists $g \in G(k)$ such that ${ }^{g} \mathcal{Z}$ admits a $W$-frame.

(3) Similarly, for any cocharacter $\mu$ of $G$, there exists a $G(k)$-conjugate $\mu^{\prime}$ of $\mu$ such that $\mathcal{Z}_{\mu^{\prime}}$ admits a $W$-frame.

(4) Let $(B, T, z)$ be a $W$-frame. For $w \in W$, choose a representative $\dot{w} \in N_{G}(T)$, such that $\left(w_{1} w_{2}\right)^{\cdot}=\dot{w}_{1} \dot{w}_{2}$ whenever $\ell\left(w_{1} w_{2}\right)=\ell\left(w_{1}\right)+\ell\left(w_{2}\right)$ (this is possible by choosing a Chevalley system, [ABD ${ }^{+} 66$, XXIII, $\left.\S 6\right]$ ). Then $\left({ }^{z} B, T, \dot{z}^{-1}\right)$ is a frame as defined in [PWZ11, Def. 3.6].

(5) If $(B, T, z)$ is a $W$-frame, then condition (iv) of Def. 1.3.1 implies $z \in W^{J}$.

Definition 1.3.3. Let $(B, T)$ be a Borel pair and $z \in W$. We call $(B, T, z)$ a dual $W$-frame for $\mathcal{Z}$ if the following conditions are satisfied:

(i) $B, T$ are defined over $\mathbf{F}_{p}$.

(ii) $B \subset Q$.

(iii) ${ }^{z} B \subset P$.

(iv) $\varphi\left({ }^{z} B \cap L\right)=B \cap M$.

The convention $B \subset P$ was used in [GKb], contrary to [PWZ11] and [PWZ15], who use the convention $B \subset Q$. The advantage of our definition of $W$-frame is that the parabolic $P$ exists naturally over the integral model of $G$, in the context of Shimura varieties. However, the result on Hasse invariants (Th. 4.1.7) can be stated more easily for a dual $W$-frame. 
Let $(G, \mu)$ a cocharacter datum and denote by $\mathcal{Z}_{\mu}=\left(G, P, L, Q, M, \varphi^{n}\right)$ its associated zip datum of exponent $n$. Fix a Borel pair $(B, T)$ defined over $\mathbf{F}_{p}$ such that $B \subset P$. We leave the following easy lemma to the reader:

Lemma 1.3.4. The triple $\left(B, T, w_{0} w_{0, J}\right)$ is a $W$-frame for the zip datum $\mathcal{Z}_{\mu}$.

1.4. Maximal cocharacter data. Let $(G, \mu)$ be a cocharacter datum over $\mathbf{F}_{p}$. We say that $(G, \mu)$ is maximal if there exists a finite-dimensional $\mathbf{F}_{p}$-vector space $V$ and an $\mathbf{F}_{p}$-representation with central (scheme-theoretic) kernel

$$
r: G \rightarrow G L(V)
$$

such that $r_{k} \circ \mu: \mathbf{G}_{m, k} \rightarrow G L(V)_{k}$ has exactly two weights. Equivalently, there is a morphism of cocharacter data with central kernel $(G, \mu) \rightarrow\left(G L(V), \mu^{\prime}\right)$ such that $\mu^{\prime}$ defines a maximal parabolic subgroup in $G L(V)_{k}$.

Proposition 1.4.1. Let $(G, \mu)$ be a cocharacter datum over $\mathbf{F}_{p}$. The following assertions are equivalent:

(1) $(G, \mu)$ is maximal.

(2) There exists a finite-dimensional k-vector space $W$ and a representation $r^{\prime}: G_{k} \rightarrow G L(W)$ over $k$ with central kernel such that $r^{\prime} \circ \mu$ has only two weights.

Proof. Clearly (11) implies (2). Conversely, let $r^{\prime}: G_{k} \rightarrow G L(W)$ be a representation as in (2). Choosing a basis of $W$, we obtain a representation $r^{\prime}: G_{k} \rightarrow G L_{m, k}$. There exists $d \geq 1$ such that $r^{\prime}$ is defined over $\mathbf{F}_{q}$ where $q=p^{d}$, hence $r^{\prime}$ comes from a morphism $r_{1}: G_{\mathbf{F}_{q}} \rightarrow G L_{m, \mathbf{F}_{q}}$. Then the representation

$$
r:=\bigoplus_{\sigma \in \operatorname{Gal}\left(\mathbf{F}_{q} / \mathbf{F}_{p}\right)}{ }^{\sigma} r_{1}
$$

is defined over $\mathbf{F}_{p}$, so it comes from a morphism $r: G \rightarrow G L_{m^{\prime}, \mathbf{F}_{p}}$ and it is clear that $r \circ \mu$ has only two weights.

Corollary 1.4.2. Let $(G, \mu)$ be a maximal cocharacter datum over $\mathbf{F}_{p}$. Let $H$ be a reductive group over $\mathbf{F}_{p}$ and $\theta: G_{k} \rightarrow H_{k}$ an isomorphism. Then $(H, \theta \circ \mu)$ is maximal.

Let $(G, \mu)$ be maximal and let $r: G \rightarrow G L(V)$ over $\mathbf{F}_{p}$ such that $\mu^{\prime}:=r_{k} \circ \mu$ has exactly two weights. Let $P \subset G_{k}$ denote the parabolic attached to $(G, \mu)$ by $\$ 1.2$. Similarly, write $P_{+}^{\prime}, P_{-}^{\prime} \subset G L(V)_{k}$ for the opposite parabolic subgroups attached to $\mu^{\prime}$, as in $\$ 1.2$. Then $P_{-}^{\prime}$ (resp. $\left.P_{+}^{\prime}\right)$ is the stabilizer of a unique subspace $V_{-} \subset V_{k}$ (resp. $V_{+} \subset V_{k}$ ), and $V_{k}=V_{-} \oplus V_{+}$. Define a character $\chi^{\prime}=\chi^{\prime}(\mu, r) \in X^{*}\left(P_{-}^{\prime}\right)$ by $\chi^{\prime}(x)=\operatorname{det}\left(x \mid V_{-}\right)$.

Definition 1.4.3. Let $(G, \mu)$ be a maximal cocharacter datum over $\mathbf{F}_{p}$ and $\chi \in X^{*}(P)$. We say that $\chi$ is maximal if there exists $r:(G, \mu) \rightarrow\left(G L(V), \mu^{\prime}\right)$ as above such that $\chi=\left.\chi^{\prime}\right|_{P}$.

Remark 1.4.4. A Hodge-type zip datum ([GKb, Def. 1.3.1]) is maximal.

1.5. Stack of $G$-zips. Let $\mathcal{Z}:=\left(G, P, Q, L, M, \varphi^{n}\right)$ be a zip datum of exponent $n$. Recall the following definition:

Definition 1.5.1 ([PWZ15, Def. 3.1]). A G-zip of type $\mathcal{Z}$ over a $k$-scheme $S$ is a tuple $\underline{\mathcal{I}}=\left(\mathcal{I}, \mathcal{I}_{P}, \mathcal{I}_{Q}, \iota\right)$ where $\mathcal{I}$ is a $G$-torsor over $S, \mathcal{I}_{P} \subset \mathcal{I}$ is a P-torsor over $S, \mathcal{I}_{Q} \subset \mathcal{I}$ is a $Q$-torsor over $S$, and $\iota:\left(\mathcal{I}_{P}\right)^{\left(p^{n}\right)} / R_{u}(P)^{\left(p^{n}\right)} \rightarrow$ $\mathcal{I}_{Q} / R_{u}(Q)$ an isomorphism of $M$-torsors.

The category of $G$-zips of type $\mathcal{Z}$ over $S$ is denoted by $G$-Zip ${ }^{\mathcal{Z}}(S)$. The $G$-Zip ${ }^{\mathcal{Z}}(S)$ give rise to a fibered category $G$-Zip ${ }^{\mathcal{Z}}$ over the category of $k$-schemes, which is a smooth stack of dimension 0 ([PWZ15, Th. 1.5]).

We denote by $x \mapsto \bar{x}$ the natural projections $P \rightarrow L$ and $Q \rightarrow M$ given by reduction modulo the unipotent radical. The zip group is a subgroup of $P \times Q$ defined by:

$$
E_{\mathcal{Z}}:=\left\{(x, y) \in P \times Q, \varphi^{n}(\bar{x})=\bar{y}\right\} .
$$

The group $G \times G$ acts naturally on $G$ via $(a, b) \cdot g:=a g b^{-1}$, and we let $P \times Q$ and $E$ act on $G$ by restricting this action. We can decompose $E_{\mathcal{Z}}$ as a semi-direct product:

$$
(U \times V) \rtimes L \simeq E_{\mathcal{Z}}, \quad((u, v), x) \longmapsto(x u, \varphi(x) v) .
$$

Recall the following theorem:

Theorem 1.5.2 ([PWZ15], Prop. 3.11). The stack of $G$-zips of type $\mathcal{Z}$ is isomorphic to the quotient stack $\left[E_{\mathcal{Z}} \backslash G\right]$.

Lemma 1.5.3. The above construction gives rise to a functor

$$
\Re_{n}: \mathscr{D}_{n} \longrightarrow(k-\text { Stacks }), \mathcal{Z} \mapsto G \text {-Zip }{ }^{\mathcal{Z}} .
$$

Proof. Let $\left(G_{1}, P_{1}, L_{1}, Q_{1}, M_{1}, \varphi^{n}\right) \rightarrow\left(G_{2}, P_{2}, L_{2}, Q_{2}, M_{2}, \varphi^{n}\right)$ be a morphism of exponent $n$ zip data. Define

$$
\alpha: G_{1} \times E_{1} \rightarrow E_{2},\left(g_{1}, p_{1}, q_{1}\right) \mapsto\left(f\left(p_{1}\right), f\left(q_{1}\right)\right) .
$$

The fact that $f$ is a morphism of zip data ensures that $\alpha$ is well-defined. Conditions (1.1.1) and (1.1.2) of $\S 1.1$ in GKb] are satisfied, hence $(f, \alpha)$ induces a morphism of stacks $\left[E_{1} \backslash G_{1}\right] \rightarrow\left[E_{2} \backslash G_{2}\right]$. 
By composition, we obtain a family of functors $\Re_{n} \circ \mathcal{Z}_{n}$ from $\mathscr{D}^{\text {co }}$ to the 2-category of $k$-stacks. We call the stack attached in this way to the cocharacter datum $(G, \mu)$ the stack of $G$-zips of type $\mu$ (and exponent $n$ ), and we denote it by $G$-Zip ${ }_{n}^{\mu}$. For $n=1$, we denote it simply by $G$-Zip ${ }^{\mu}$.

\section{Proposition 1.5.4.}

(1) Let $\mu$ be a cocharacter of $G_{k}$ and $\mu^{\prime}=\operatorname{ad}(g) \circ \mu$ for some $g \in G(k)$. Then the $k$-stacks $G$-Zip ${ }_{n}^{\mu}$ and $G$-Zip ${ }_{n}^{\mu^{\prime}}$ are isomorphic.

(2) More generally, let $\mathcal{Z}=\left(G, P, L, Q, M, \varphi^{n}\right)$ be a zip datum, and $g \in G(k)$. Then the $k$-stacks $G$-Zip ${ }^{\mathcal{Z}}$ and $G$-Zip ${ }^{g} \mathcal{Z}$ are isomorphic.

Proof. It is easy to check that the map $G \rightarrow G$ given by $x \mapsto g x \varphi^{n}(g)^{-1}$ induces an isomorphism of stacks.

1.6. Stratification. We recall the results of [PWZ11], but reformulated using our convention (Def. 1.3.1). Choose a system of representatives $\dot{w} \in G$ for $w \in W$ as in Rmk. 1.3.2(4).

Let $\mathcal{Z}=\left(G, P, L, Q, M, \varphi^{n}\right)$ be a zip datum, and let $(B, T, z)$ be a $W$-frame of $\mathcal{Z}$. If $h \in G(k)$ is an element of the group, we denote by $\mathcal{O}_{\mathcal{Z}}(h)$ the $E$-orbit of $h$ in $G$. Similarly, we write $o_{\mathcal{Z}}(h):=\left[E_{\mathcal{Z}} \backslash \mathcal{O}_{\mathcal{Z}}(h)\right]$ for the corresponding $k$-point in $\left[E_{\mathcal{Z}} \backslash G\right]$. For $w \in W$, define $G_{w}:=\mathcal{O}_{\mathcal{Z}}\left(\dot{w} \dot{z}^{-1}\right)$.

By Ths. 7.5, 11.3 in loc. cit., the map $w \mapsto G_{w}$ restricts to two bijections

$$
\begin{aligned}
{ }^{I} W & \rightarrow\left\{E_{\mathcal{Z}} \text {-orbits in } G\right\} \\
W^{J} & \rightarrow\left\{E_{\mathcal{Z}} \text {-orbits in } G\right\}
\end{aligned}
$$

Furthermore, one has the following dimension formulas:

$$
\operatorname{dim}\left(G_{w}\right)=\ell(w)+\operatorname{dim}(P) \quad \text { for all } w \in{ }^{I} W \cup W^{J} .
$$

\section{THE STACK OF ZIP FLAGS}

Let $\mathcal{Z}=\left(G, P, L, Q, M, \varphi^{n}\right)$ be a zip datum and set $q:=p^{n}$. We assume that $\mathcal{Z}$ admits a $\mathrm{W}$-frame $(B, T, z)$, which we fix (this assumption is harmless by Rmk. 1.3.2 (2) and Prop. 1.5.4). As in subsection [1.3, we denote by $\Phi, \Phi^{+}, \Delta$ the roots, positive roots, simple roots with respect to $(B, T)$ respectively, and we denote by $I, J \subset \Delta$ the types of $P, Q$ respectively.

2.1. Definition of the stack $G$-ZipFlag ${ }^{\left(\mathcal{Z}, P_{0}\right)}$. We denote by $P_{0}$ a parabolic subgroup such that $B \subset P_{0} \subset P$, and we let $I_{0} \subset I$ denote the type of $P_{0}$. We call $\left(\mathcal{Z}, P_{0}\right)$ a flagged zip datum.

Definition 2.1.1. A G-zip flag of type $\left(\mathcal{Z}, P_{0}\right)$ and exponent $n$ over a $k$-scheme $S$ is a pair $\hat{\mathcal{I}}=(\underline{\mathcal{I}}, \mathcal{J})$ where $\underline{\mathcal{I}}=\left(\mathcal{I}, \mathcal{I}_{P}, \mathcal{I}_{Q}, \iota\right)$ is a $G$-zip of type $\mathcal{Z}$ and exponent $n$ over $S$, and $\mathcal{J} \subset \mathcal{I}_{P}$ is a $P_{0}$-torsor.

We denote by $G$-ZipFlag ${ }^{\left(\mathcal{Z}, P_{0}\right)}(S)$ the category of $G$-zip flags over $S$ of type $\left(\mathcal{Z}, P_{0}\right)$. By the same arguments as for $G$-zips, we obtain a stack $G$-ZipFlag ${ }^{\left(\mathcal{Z}, P_{0}\right)}$ over $k$, which we call the stack of $G$-zip flags of type $\left(\mathcal{Z}, P_{0}\right)$. There is a natural morphism of stacks

$$
\pi_{P_{0}}: G \text {-ZipFlag }{ }^{\left(\mathcal{Z}, P_{0}\right)} \longrightarrow G \text {-Zip }{ }^{\mathcal{Z}}
$$

which forgets the $P_{0}$-torsor $J$ on the level of categories $G$-ZipFlag ${ }^{\left(\mathcal{Z}, P_{0}\right)}(S) \rightarrow G$-Zip ${ }^{\mathcal{Z}}(S)$. More generally, for each pair of parabolics $\left(P_{0}, P_{1}\right)$ such that $B \subset P_{1} \subset P_{0} \subset P$, we have a natural map of stacks

$$
\pi_{P_{1}, P_{0}}: G \text {-ZipFlag }{ }^{\left(\mathcal{Z}, P_{1}\right)} \longrightarrow G \text {-ZipFlag }{ }^{\left(\mathcal{Z}, P_{0}\right)} \text {. }
$$

Note that when $P_{0}=P$, the stack $G$-ZipFlag ${ }^{(\mathcal{Z}, P)}$ identifies with $G$-Zip ${ }^{\mathcal{Z}}$ through the isomorphism $\pi_{P}$. Hence we can identify the maps $\pi_{P_{0}}$ and $\pi_{P_{0}, P}$. We obtain a tower of stacks above $G$-Zip ${ }^{\mathcal{Z}}$. Define a subgroup $\hat{E}_{P_{0}} \subset E_{\mathcal{Z}}$ by:

$$
\hat{E}_{P_{0}}:=\left\{(x, y) \in E_{\mathcal{Z}}, x \in P_{0}\right\} .
$$

To avoid confusion, we will also denote the group $\hat{E}_{P_{0}}$ by $\hat{E}_{P_{0}}^{\mathcal{Z}}$ to emphasize that it depends on the zip datum $\mathcal{Z}$.

\section{Theorem 2.1.2.}

(1) The stack $G$-ZipFlag ${ }^{\left(\mathcal{Z}, P_{0}\right)}$ is a smooth stack of dimension $\operatorname{dim}\left(P / P_{0}\right)$, isomorphic to $\left[\hat{E}_{P_{0}} \backslash G\right]$.

(2) For all parabolics $B \subset P_{1} \subset P_{0} \subset P$, we have a commutative diagram

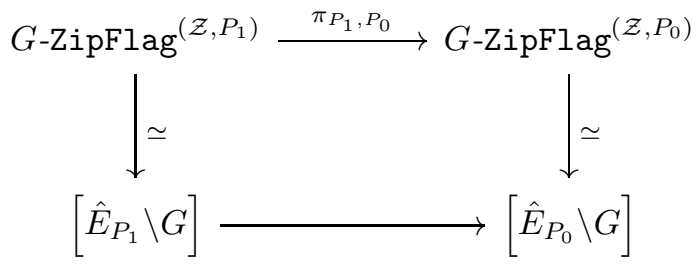


where the vertical maps are isomorphisms and the lower horizontal map is induced by the natural projection.

(3) The map $\pi_{P_{1}, P_{0}}$ is proper and smooth, with fibers isomorphic to $P_{0} / P_{1}$.

Proof. The proof is entirely similar to the proof of [GKb, Th. 5.1.3]. The assertion on the dimension follows from (3) and $\operatorname{dim}\left(G-\right.$ Zip $\left.^{\mathcal{Z}}\right)=0$.

In other words, (2) states that there is an isomorphism of towers $G$-ZipFlag ${ }^{\left(\mathcal{Z}, P_{\bullet}\right)} \simeq\left[\hat{E}_{P_{\bullet}} \backslash G\right]$. We set $P_{0, L}:=$ $P_{0} \cap L$, and similarly $B_{L}:=B \cap L$. The inclusion $G \rightarrow G \times L, g \mapsto(g, 1)$ induces an isomorphism of quotient stacks

$$
\left[\hat{E}_{P_{0}} \backslash G\right] \longrightarrow\left[E \backslash\left(G \times L / P_{0, L}\right)\right] .
$$

In this description, the same group $E$ acts at all levels of the tower, and the natural projections are $E$-equivariant.

2.2. Coarse flag strata. We identify without explicit mention the stack of zip flags of type $\left(\mathcal{Z}, P_{0}\right)$ and the quotient stack $\left[\hat{E}_{P_{0}} \backslash G\right]$ (Th. 2.1.2). Define a parabolic subgroup $Q_{0}$ by

$$
Q_{0}:=\left(L \cap P_{0}\right)^{\left(p^{n}\right)} R_{u}(Q)
$$

Note that ${ }^{z} B \subset Q_{0} \subset Q$ and $\hat{E}_{P_{0}} \subset P_{0} \times Q_{0}$. Denote by $J_{0} \subset J$ the type of $Q_{0}$. Hence we have a natural smooth surjective morphism of stacks:

$$
\psi_{P_{0}}: G-\operatorname{ZipFlag}\left(\mathcal{Z}, P_{0}\right)=\left[\hat{E}_{P_{0}} \backslash G\right] \rightarrow\left[\left(P_{0} \times Q_{0}\right) \backslash G\right] .
$$

We define the coarse flag strata of the stack $G$-ZipFlag $\left(\mathcal{Z}, P_{0}\right)$ as the fibers of the morphism $\psi_{P_{0}}$ (endowed with the reduced structure). Hence the coarse flag strata are the quotient stacks $\left[\hat{E}_{P_{0}} \backslash C\right]$ for $C$ a $P_{0} \times Q_{0}$-orbit in $G$. Define a closed coarse flag stratum as the preimage of the closure of a point in $\left[\left(P_{0} \times Q_{0}\right) \backslash G\right]$ by $\psi_{P_{0}}$ (endowed with the reduced structure). Using the isomorphism (2.1.4), we obtain a map

$$
\left[E \backslash\left(G \times L / P_{0, L}\right)\right] \rightarrow\left[\left(P_{0} \times Q_{0}\right) \backslash G\right] .
$$

which is induced by the map: $\tilde{\psi}_{P_{0}}: G \times L \rightarrow G, \quad(g, x) \mapsto x^{-1} g \varphi^{n}(x)$.

Recall that the underlying topological space of $\left[\left(P_{0} \times Q_{0}\right) \backslash G\right]$ is isomorphic to ${ }^{I_{0}} W^{J_{0}}$ endowed with the topology attached to the Bruhat order. The parametrization is given by the map:

$$
{ }^{I_{0}} W^{J_{0}} \rightarrow\left[\left(P_{0} \times Q_{0}\right) \backslash G\right], \quad w \mapsto P_{0} \dot{w} \dot{z}^{-1} Q_{0} .
$$

For $w \in{ }^{I_{0}} W^{J_{0}}$, we denote by $C_{P_{0}, w}:=P_{0} \dot{w} \dot{z}^{-1} Q_{0}$ the corresponding $P_{0} \times Q_{0}$-orbit, and define

$$
\mathbf{Z}_{P_{0}, w}:=\left[\hat{E}_{P_{0}} \backslash C_{P_{0}, w}\right]
$$

the corresponding coarse flag stratum. Similarly, using the description given by isomorphism (2.1.4), we also define:

$$
\mathbf{H}_{P_{0}, w}:=\left\{(g, x) \in G \times L / P_{0, L}, x^{-1} g \varphi^{n}(x) \in C_{P_{0}, w}\right\} .
$$

Thus $\mathbf{H}_{P_{0}, w}$ is a locally closed $E$-stable subvariety of $G \times L / P_{0, L}$ and $\mathbf{Z}_{P_{0}, w}=\left[E \backslash \mathbf{H}_{P_{0}, w}\right]$.

\section{Proposition 2.2.1.}

(1) The coarse flag strata are irreducible, smooth, and their closures are normal.

(2) The coarse closed flag strata coincide with the closures of coarse flag strata.

(3) Set $I_{w}:=J_{0} \cap w^{-1} I_{0} w$. One has $\operatorname{dim}\left(\mathbf{H}_{P_{0}, w}\right)=\ell(w)+\ell\left(w_{0, J_{0}}\right)-\ell\left(w_{0, I_{w}}\right)-\operatorname{dim}\left(P_{0}\right)$.

Proof. The first two parts are a simple generalization of Th. 5.3.3 in GKb. The third one is a consequence of Prop. 1.12 in Wed14.

For parabolics $B \subset P_{1} \subset P_{0}$ with corresponding subgroups ${ }^{z} B \subset Q_{1} \subset Q_{0}$, we have a commutative diagram



where the rightmost vertical map is the natural projection. Hence the maps $\left(\psi_{P_{0}}\right)_{P_{0}}$ define a morphism of towers

$$
\psi: G \text {-ZipFlag }{ }^{\left(\mathcal{Z}, P_{\bullet}\right)} \rightarrow\left[\left(P_{\bullet} \times Q_{\bullet}\right) \backslash G\right] .
$$

\section{Fine flag Strata}

In this section, we define a stratification of $G$-ZipFlag ${ }^{\left(\mathcal{Z}, P_{0}\right)}$ which is finer that the stratification given by $\psi_{P_{0}}$. 
3.1. Definition of fine flag strata. For a parabolic $B \subset P_{0} \subset P$ with type $I_{0} \subset I$, we always denote by $Q_{0}$ the parabolic attached to $P_{0}$ by formula (2.2.1) and by $J_{0}$ its type. Let $L_{0}$ (resp. $M_{0}$ ) be the Levi subgroup of $P_{0}$ (resp. $Q_{0}$ ) containing $T$. It follows from the definition that $\varphi^{n}\left(L_{0}\right)=M_{0}$. Hence the tuple

$$
\mathcal{Z}_{0}:=\left(G, P_{0}, L_{0}, Q_{0}, M_{0}, \varphi^{n}\right)
$$

is a zip datum of exponent $n$. We obtain a stack $G$-Zip ${ }^{\mathcal{Z}_{0}}$ and a zip group $E_{\mathcal{Z}_{0}}$ such that $\hat{E}_{P_{0}} \subset E_{\mathcal{Z}_{0}} \subset P_{0} \times Q_{0}$. Hence we obtain a smooth surjective morphism of stacks

$$
\Psi_{P_{0}}: G \text {-ZipFlag }{ }^{\left(\mathcal{Z}, P_{0}\right)} \rightarrow G-\text { Zip }^{\mathcal{Z}_{0}} \simeq\left[E_{\mathcal{Z}_{0}} \backslash G\right]
$$

which induces a factorization of $\psi_{P_{0}}$ :

$$
G \text {-ZipFlag }\left(\mathcal{Z}, P_{0}\right) \stackrel{\Psi_{P_{0}}}{\longrightarrow} G \text {-Zip } \text { Z }^{\mathcal{Z}_{0}} \simeq\left[\hat{E}_{P_{0}} \backslash G\right] \stackrel{\beta}{\rightarrow}\left[\left(P_{0} \times Q_{0}\right) \backslash G\right]
$$

where $\beta$ is the natural projection. Define fine flag strata as the fibers of the map $\Psi_{P_{0}}$ (endowed with the reduced structure). Fine flag strata coincide with coarse flag strata when $P_{0}=B$ and with usual zip strata when $P_{0}=P$.

We can decompose the groups $E_{\mathcal{Z}_{0}}$ and $\hat{E}_{P_{0}}$ as semi-direct products:

$$
\begin{aligned}
& \left(U_{0} \times V_{0}\right) \rtimes L_{0} \simeq E_{\mathcal{Z}_{0}}, \quad((u, v), x) \longmapsto(x u, \varphi(x) v) \\
& \left(U_{0} \ltimes V\right) \rtimes L_{0} \simeq \hat{E}_{P_{0}}, \quad((u, v), x) \longmapsto\left(x u, \varphi\left(x \theta_{L}^{P}(u)\right) v\right)
\end{aligned}
$$

where $\theta_{L}^{P}: P \rightarrow L$ denotes the reduction modulo the unipotent radical $U$.

Lemma 3.1.1. One has an isomorphism $E_{\mathcal{Z}_{0}} / \hat{E}_{P_{0}} \simeq M \cap V_{0} \simeq V_{0} / V \simeq \mathbf{A}^{\operatorname{dim}\left(P / P_{0}\right)}$.

Proof. Using (3.1.4) and (3.1.5), one sees that the map $V_{0} \rightarrow E_{\mathcal{Z}_{0}} / \hat{E}_{P_{0}}$ taking $u \in V_{0}$ to the class of $(1, u) \in E_{\mathcal{Z}_{0}}$ is surjective, and induces an isomorphism $V_{0} / V \simeq E_{\mathcal{Z}_{0}} / \hat{E}_{P_{0}}$. It is also clear that the inclusion $M \cap V_{0} \subset V_{0}$ induces an isomorphism $M \cap V_{0} \simeq V_{0} / V$. Since $M \cap V_{0}$ is unipotent, it is isomorphic as a variety to $\mathbf{A}^{r}$, with $r=\operatorname{dim}\left(M \cap V_{0}\right)=\operatorname{dim}\left(P / P_{0}\right)$.

For parabolic subgroups $B \subset P_{1} \subset P_{0} \subset P$, one has inclusions

$$
\begin{array}{ccccc}
\hat{E}_{P_{1}} & \subset & \hat{E}_{P_{0}} \\
\cap & & \cap \\
E_{\mathcal{Z}_{1}} & & & E_{\mathcal{Z}_{0}}
\end{array}
$$

but in general there is no inclusion between $E_{\mathcal{Z}_{0}}$ and $E_{\mathcal{Z}_{1}}$.

Proposition 3.1.2. Let $B \subset P_{1} \subset P_{0} \subset P$ be parabolic subgroups. Then the map $\pi_{P_{1}, P_{0}}$ sends a fine flag stratum for $P_{1}$ to a union of fine flag strata for $P_{0}$.

Proof. Denote by $\mathcal{Z}_{1}$ the zip datum attached to $P_{1}$ as in (3.1.1). If $C$ is an $E_{\mathcal{Z}_{1}}$-orbit in $G$, we must show that the set $X:=\hat{E}_{P_{0}} \cdot C$ is $E_{\mathcal{Z}_{0}}$-stable. Since $X$ is clearly stable by $\varphi^{n}$-conjugation by $L_{0}$, it suffices to show that $X$ is stable by elements $(u, v) \in R_{u} P_{0} \times R_{u} Q_{0}$. Let $x \in X$ be an element. Since $R_{u} P \times R_{u} Q \subset \hat{E}_{P_{0}}$, we may assume that there exists $a \in P_{0, L}$ such that $y:=a x \varphi^{n}(a)^{-1}$ is in $C$. Write $a=a^{\prime} r$ for $a^{\prime} \in L_{0}$ and $r \in R_{u} P_{0}$. Then

$$
u x v=u a^{-1} y \varphi^{n}(a) v=a^{\prime-1} u^{\prime} y v^{\prime} \varphi^{n}\left(a^{\prime}\right)
$$

for some elements $\left(u^{\prime}, v^{\prime}\right) \in R_{u} P_{0} \times R_{u} Q_{0}$. Since $R_{u} P_{0} \times R_{u} Q_{0} \subset R_{u} P_{1} \times R_{u} Q_{1}$, we deduce that $u^{\prime} y v^{\prime} \in C$, and hence $u x v \in X$ as claimed.

For a parabolic $B \subset P_{0} \subset P$ with type $I_{0} \subset I$ and $w \in W$, define

$$
Z_{P_{0}, w}:=\Psi_{P_{0}}^{-1}\left(o \mathcal{Z}_{0}\left(\dot{w} \dot{z}^{-1}\right)\right)
$$

endowed with the reduced structure as locally closed substack. Similarly to the parametrizations (1.6.1) and (1.6.2) for $E$-orbits in $G$, the map $w \mapsto Z_{P_{0}, w}$ restricts to two bijections

$$
\begin{aligned}
& { }^{I_{0}} W \longrightarrow\left\{\text { fine flag strata of } G \text {-ZipFlag }{ }^{\left(\mathcal{Z}, P_{0}\right)}\right\} \\
& W^{J_{0}} \longrightarrow\left\{\text { fine flag strata of } G \text {-ZipFlag }{ }^{\left(\mathcal{Z}, P_{0}\right)}\right\} \text {. }
\end{aligned}
$$

For $w \in{ }^{I_{0}} W \cup W^{J_{0}}$, define a locally closed subvariety of $G \times\left(L / P_{0, L}\right)$ by

$$
H_{P_{0}, w}:=\left\{(g, x) \in G \times L / P_{0, L}, x^{-1} g \varphi^{n}(x) \in \mathcal{O}_{\mathcal{Z}_{0}}\left(\dot{w} \dot{z}^{-1}\right)\right\} .
$$

It is $E$-stable and one has $Z_{P_{0}, w}=\left[E \backslash H_{P_{0}, w}\right]$.

Proposition 3.1.3. For $w \in{ }^{I_{0}} W \cup W^{J_{0}}$, the variety $H_{P_{0}, w}$ (resp. the stack $Z_{P_{0}, w}$ ) is smooth of dimension $\ell(w)+\operatorname{dim}(P)$ (resp. $\ell(w)+\operatorname{dim}(P)-\operatorname{dim}(G))$. 
Proof. This follows immediately from Prop. 3.1.1 and the dimension formula (1.6.3).

If different zip data come into play, we write a superscript indicating the zip datum one considers. For example, consider parabolics $B \subset P_{1} \subset P_{0} \subset P$. We have defined a zip datum $\mathcal{Z}_{0}=\left(G, P_{0}, L_{0}, Q_{0}, M_{0}, \varphi^{n}\right)$. Hence it makes sense to speak of $G$-zip flags of type $\left(\mathcal{Z}_{0}, P_{1}\right)$. Therefore we define $\pi_{P_{1}}^{\mathcal{Z}_{0}}$ and $\Psi_{P_{1}}^{\mathcal{Z}_{0}}$ as the maps attached to $P_{1}$ with respect to the zip datum $\mathcal{Z}_{0}$ by (2.1.2) and (3.1.2) respectively.

Proposition 3.1.4. Let $B \subset P_{1} \subset P_{0} \subset P$ be parabolic subgroups. There is a natural map

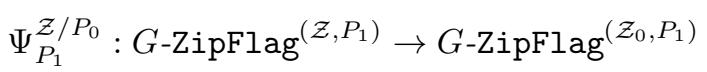

such that the following diagram commutes:

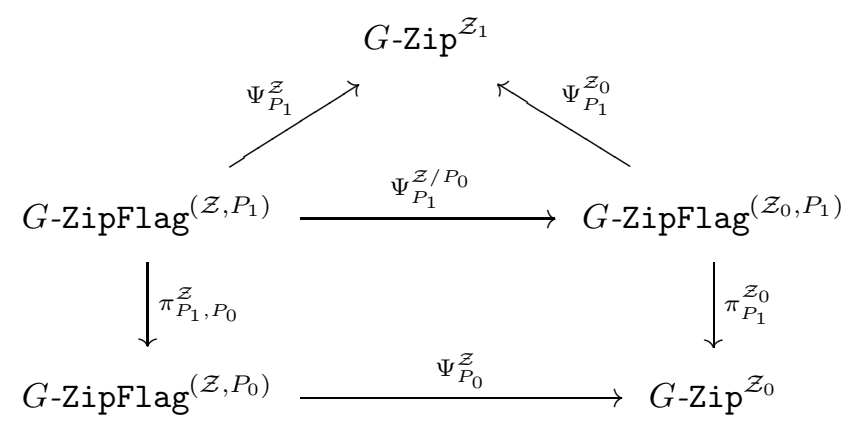

Moreover, the commutative square above is cartesian.

Proof. This follows simply from the bicartesian square



We mean by this that the subgroups $\hat{E}_{P_{1}}^{\mathcal{Z}_{0}}$ and $\hat{E}_{P_{0}}^{\mathcal{Z}}$ generate $E_{\mathcal{Z}_{0}}$ and their intersection is exactly $\hat{E}_{P_{1}}^{\mathcal{Z}}$.

Remark 3.1.5. The morphism $\Psi_{P_{1} / P_{0}}^{\mathcal{Z}}$ induces thus a bijection between fine flag strata of $G$-ZipFlag ${ }^{\left(\mathcal{Z}, P_{1}\right)}$ and $G$-ZipFlag $\left(\mathcal{Z}_{0}, P_{1}\right)$. Hence, any statement stable by base change regarding intermediate parabolics $P_{1} \subset P_{0}$ can be reduced to a result involving just the parabolic $P_{1}$, by changing the zip datum to $\mathcal{Z}_{0}$.

Prop. 3.1.4 can be generalized as follows. The proof is similar, so we skip it. Consider four parabolics $B \subset P_{\square} \subset$ $P_{\boldsymbol{}} \subset P_{\diamond} \subset P_{\diamond} \subset P$. There is a commutative diagram with a cartesian square in the middle:

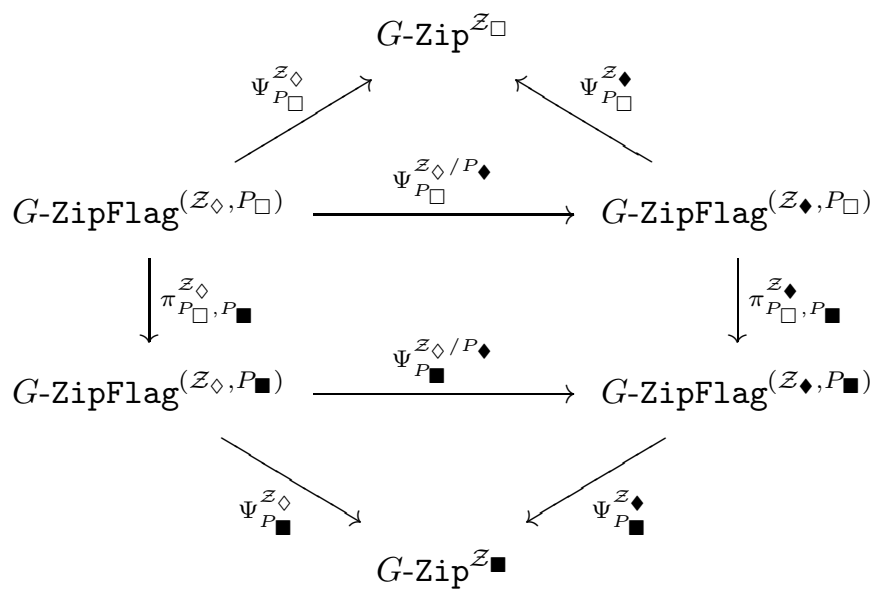

3.2. Minimal, cominimal fine flag strata. In general, it is difficult to determine the image of a fine flag stratum by $\pi_{P_{1}, P_{0}}$. In this section we give a partial result.

Definition 3.2.1. Let $B \subset P_{1} \subset P_{0} \subset P$ be parabolics of type $I_{1} \subset I_{0} \subset I$. A $P_{0}$-minimal (resp. $P_{0}$-cominimal) fine flag stratum of $G$-ZipFlag ${ }^{\left(\mathcal{Z}, P_{1}\right)}$ is a stratum of the form $Z_{P_{1}, w}$ for $w \in{ }^{I_{0}} W$ (resp. $w \in W^{J_{0}}$ ). 
This generalizes the concept of minimal strata introduced by Ekedahl and Van der Geer in [EvdG09] and also studied by the authors in GKb.

Proposition 3.2.2. Let $B \subset P_{1} \subset P_{0} \subset P$ be parabolics of type $I_{1} \subset I_{0} \subset I$. For all $w \in{ }^{I_{0}} W \cup W^{J_{0}}$, one has:

$$
\pi_{P_{1}, P_{0}}\left(Z_{P_{1}, w}\right)=Z_{P_{0}, w} .
$$

Moreover, the map $\pi_{P_{1}, P_{0}}: Z_{P_{1}, w} \rightarrow Z_{P_{0}, w}$ is finite.

Proof. In the special case $P_{1}=B$ and $P_{0}=P$, the result follows from [GKb, Prop. 5.4.3]. Using Prop. 3.1.4, we obtain the result for arbitrary $P_{0}$ and $P_{1}=B$. Now, let $P_{1} \subset P_{0}$ be arbitrary. By the special case, we have in particular $\pi_{B, P_{1}}\left(Z_{B, w}\right)=Z_{P_{1}, w}$. Hence:

$$
\pi_{P_{1}, P_{0}}\left(Z_{P_{1}, w}\right)=\pi_{P_{1}, P_{0}}\left(\pi_{B, P_{1}}\left(Z_{B, w}\right)\right)=\pi_{B, P_{0}}\left(Z_{B, w}\right)=Z_{P_{0}, w}
$$

where the last equality follows again from the special case.

For the finiteness of the map $\pi_{P_{1}, P_{0}}: Z_{P_{1}, w} \rightarrow Z_{P_{0}, w}$, we reduce again to the case $P_{1}=B$, where it is known by [GKb, Prop. 5.4.3]. We use the varieties $H_{P_{0}, w}$ defined in (3.1.9) to use classical algebraic geometry. Notice that $\pi_{P_{1}, P_{0}}$ induces a surjective proper map $\tilde{\pi}_{P_{1}, P_{0}}: H_{P_{1}, w} \rightarrow H_{P_{0}, w}$, and it suffices to show that this map is quasi-finite. The map $\tilde{\pi}_{B, P_{0}}$ factors as a composition $H_{B, w} \stackrel{\tilde{\pi}_{B, P_{1}}}{\longrightarrow} H_{P_{1}, w} \stackrel{\tilde{\pi}_{P_{1}, P_{0}}}{\longrightarrow} H_{P_{0}, w}$. Since $\tilde{\pi}_{B, P_{1}}$ is surjective and the composition is quasi-finite, the second map must be quasi-finite. This concludes the proof.

3.3. Closure relations for $G$-Zip ${ }^{\mathcal{Z}}$. For $w \in{ }^{I} W$, write $G^{w}:=\mathcal{O}\left(\dot{w} \dot{z}^{-1}\right)$ as in $\$ 1.6$ Denote by $\leq$ the Bruhat order on $W$. For $w, w^{\prime} \in{ }^{I} W$, define $w \preceq w^{\prime}$ if and only if there exists $y \in W_{I}$ such that $y w^{\prime} \varphi^{n}(y)^{-1} \leq w$. Recall the following:

Theorem 3.3.1 ([PWZ11, Th. 6.2]). The Zariski closure of $G^{w}$ is the union of all $G^{w^{\prime}}$ with $w^{\prime} \preceq w$.

We show below how the flag space can be used to prove a similar result. Consider the flag space $G$-ZipFlag ${ }^{(\mathcal{Z}, B)}$, which we denote simply by $G$-ZipFlag ${ }^{\mathcal{Z}}$. Since we only consider the parabolic $P$, we shorten the term " $P$-minimal" to "minimal" and similarly for "cominimal". For elements $x, y \in G(k)$, we define two relations:

$$
\begin{aligned}
& x \leq y \Longleftrightarrow x \in \overline{B y^{z} B} \\
& x \preceq y \Longleftrightarrow x \in \overline{\mathcal{O}_{\mathcal{Z}}(y)}
\end{aligned}
$$

We say that $(g, l) \in G \times L$ is minimal (resp. cominimal) if its image by the isomorphism (2.1.4) in $G$-ZipFlag ${ }^{\mathcal{Z}}$ lies in a minimal (resp. cominimal) stratum. We say that $g \in G$ is minimal (resp. cominimal) if $(g, 1)$ is minimal (resp. cominimal).

Proposition 3.3.2. Let $x, y \in G$, with $y$ minimal or cominimal. Then:

$$
x \preceq y \Longleftrightarrow \exists h \in L, h x \varphi^{n}(h)^{-1} \leq y .
$$

Proof. We will assume that $y$ is minimal, since the proof is entirely similar in the cominimal case. Let $H \subset$ $G \times\left(L / B_{L}\right)$ denote the minimal stratum containing the image of $(y, 1)$. By Prop. 3.2.2, the image $\pi(H)$ by the projection $\pi: G \times\left(L / B_{L}\right) \rightarrow G$ is $\mathcal{O}(y)$. Now, the right-hand side of (3.3.3) amounts to the existence of $h \in L$ such that $(x, \bar{h}) \in \bar{H}$, where $\bar{h}$ is the image of $h$ by the map $L \rightarrow L / B_{L}$. But this means exactly that $x$ belongs to $\pi(\bar{H})=\overline{\mathcal{O}(y)}$, so it is equivalent to $x \preceq y$.

Remark 3.3.3. For $w \in{ }^{I} W$, the element $\dot{w} \dot{z}^{-1}$ is minimal. Applying Prop. 3.3 .2 to this element gives a similar result as [PWZ11, Th. 6.2] recalled above.

\section{Sections over fine flag strata}

4.1. Existence of sections on zip strata. We recall some results of [GKb, §3] on the existence of group-theoretical Hasse invariants. The formulas generalize readily to an arbitrary exponent $n \geq 1$.

Definition 4.1.1. Let $S$ be locally closed in a scheme (or algebraic stack) $X$, with Zariski closure $\bar{S}$ (endowed with the reduced structure), and let $\mathscr{L}$ be a line bundle on $X$. We say that a section $f \in H^{0}(\bar{S}, \mathscr{L})$ is a characteristic section for $S$ if the non-vanishing locus of $f$ in $\bar{S}$ is exactly $S$.

Fix a zip datum $\mathcal{Z}$ of exponent $n$. Assume that there exists a torus $T \subset L$ defined over $\mathbf{F}_{p}$ (we may achieve this by replacing $\mathcal{Z}$ with ${ }^{g} \mathcal{Z}$ for an appropriate $g \in G$, see Prop. 1.5.4). Identify $X^{*}(E)=X^{*}(L)$ via the maps $E \rightarrow P \rightarrow L$ (where the first map is the first projection, and the second map is the natural projection). For $\lambda \in X^{*}(L)$, denote by $\mathscr{V}(\lambda)$ the line bundle on $[E \backslash G] \simeq G$-Zip ${ }^{\mathcal{Z}}$ naturally attached to $\lambda$ by [GKb, §N.3.1].

\section{Definition 4.1.2.}


(1) Let $\chi \in X^{*}(T)$. We say that $\chi$ is orbitally $q$-close if $\left|\frac{\left\langle\chi, \sigma \alpha^{\vee}\right\rangle}{\left\langle\chi, \alpha^{\vee}\right\rangle}\right| \leq q-1$ for every $\alpha \in \Phi$ satisfying $\left\langle\chi, \alpha^{\vee}\right\rangle \neq 0$ and for all $\sigma \in W \rtimes \operatorname{Gal}\left(k / \mathbf{F}_{p}\right)$.

(2) We say that $\chi$ is $\underline{q \text {-small if }}\left|\left\langle\chi, \alpha^{\vee}\right\rangle\right| \leq q-1$ for all $\alpha \in \Phi$.

Definition 4.1.3. A character $\lambda \in X^{*}(L)$ is $\mathcal{Z}$-ample if the associated line bundle on $G / \varphi^{-n}(Q)$ is anti-ample (note that $L \subset \varphi^{-n}(Q)$ is a Levi subgroup).

Remark 4.1.4. Let $(B, T, z)$ be a $W$-frame and $\left(B^{\prime}, T^{\prime}, z^{\prime}\right)$ a dual $W$-frame. The following are equivalent:

(1) $\chi$ is $\mathcal{Z}$-ample.

(2) One has $\left\langle\chi, \varphi^{-n}(z) \alpha^{\vee}\right\rangle<0$ for all $\alpha \in \Delta \backslash \varphi^{-n}(J)$ with respect to $(B, T)$.

(3) One has $\left\langle\chi, \alpha^{\vee}\right\rangle<0$ for all $\alpha \in \Delta \backslash \varphi^{-n}(J)$ with respect to $\left(B^{\prime}, T^{\prime}\right)$.

Remark 4.1.5. Let $\mu \in X_{*}(G)$ and $\mathcal{Z}=\mathcal{Z}_{\mu}$. Let $(B, T, z)$ be a $W$-frame, $B \subset P_{0} \subset P$ a parabolic and $\mathcal{Z}_{0}$ defined by (3.1.1). The following are equivalent:

(1) $\chi$ is $\mathcal{Z}_{0}$-ample.

(2) One has $\left\langle\chi, \alpha^{\vee}\right\rangle>0$ for all $\alpha \in I \backslash I_{0}$ and $\left\langle\chi, \alpha^{\vee}\right\rangle<0$ for all $\alpha \in \Phi^{+} \backslash \Phi_{L}^{+}$.

Remark 4.1.6. Condition 4.1.5(2) is precisely the one that arises in the work of Griffiths-Schmid concerning the positivity of automorphic vector bundles on homogeneous complex manifolds, see the proof following $(4.21)_{D}$ on p. 276 of GS69]. We will elaborate on the connection between GS69] and sections on Zip strata in our forthcoming work with Brunebarbe and Stroh [BGKS.

Fix $N \geq 1$ such that $\operatorname{dim}_{k} H^{0}([E \backslash S], \mathscr{V}(N \chi))=1$ for all $E$-orbits $S \subset G$ and for all $\chi \in X^{*}(L)$ (for the existence of such $N$, see loc. cit. $\S 3)$. Denote by $h_{S, \chi}$ a nonzero element of $H^{0}([E \backslash S], \mathscr{V}(N \chi))$. Let $\bar{S}$ be the Zariski closure of $S$, endowed with the reduced structure.

Theorem 4.1.7 ([GKb, Th. 3.2.3]). If $\chi \in X^{*}(L)$ is $\mathcal{Z}$-ample and orbitally $q$-close, then there exists $d \geq 1$ such that for all E-orbit $S \subset G$, the section $\left(h_{S, \chi}\right)^{d}$ extends to $[E \backslash \bar{S}]$ with non-vanishing locus $[E \backslash S]$.

One can state a more precise result. Let $\sigma: k \rightarrow k$ denote the inverse of the map $x \mapsto x^{p}$. For $w \in W$, define $w^{(0)}=e$ and by induction $w^{(r)}:={ }^{\sigma}\left(w^{(r-1)} w\right)$ for all $r \geq 1$.

(i) Fix a $W$-frame $(B, T, z)$ (Def. 1.3.1).

(ii) Fix $m \geq 1$ such that $T$ splits over $\mathbf{F}_{q^{m}}$, where $q:=p^{n}$.

(iii) For $w \in{ }^{I} W \cup W^{J}$, choose $r_{w} \geq 1$ such that $\left(w \varphi^{n}(z)\right)^{\left(r_{w}\right)}=e$.

(iv) For $w \in W$, define $E_{w}$ as the set of roots $\alpha \in \Phi^{+}$such that $w s_{\alpha}<w$ and $\ell\left(w s_{\alpha}\right)=\ell(w)-1$.

(v) For $w \in{ }^{I} W \cup W^{J}$, denote by $h_{w, \chi}$ the section $h_{S, \chi}$ for $S=G_{w}$.

Proposition 4.1.8 ([GKb, Prop. 3.2.1]). Let $w \in{ }^{I} W \cup W^{J}$ and $\chi \in X^{*}(L)$. The following assertions are equivalent:

(1) There exists $d \geq 1$ such that $\left(h_{w, \chi}\right)^{d}$ extends to $\left[E \backslash \bar{G}_{w}\right]$ with non-vanishing locus $\left[E \backslash G_{w}\right]$ (i.e to a characteristic section of $\left.\left[E \backslash \bar{G}_{w}\right]\right)$.

(2) For all $\alpha \in E_{w}$, one has:

$$
n_{\alpha}:=\sum_{i=0}^{r_{w} m-1}\left\langle\left(z w^{-1}\right)^{(i)}\left(\sigma^{i} \chi\right), w \alpha^{\vee}\right\rangle p^{i}>0 .
$$

Corollary 4.1.9. Let $\mathcal{Z}=\left(G, P, L, Q, M, \varphi^{n}\right)$ be an exponent $n$ zip datum such that $G_{k} \simeq G L_{n, k}$ and $P$ is a maximal parabolic. For a $\mathcal{Z}$-ample character $\lambda \in X^{*}(L)$, there exists $N \geq 1$ such that $\mathscr{V}(N \lambda)$ admits characteristic sections on all zip strata.

Proof. It is clear that $\lambda$ is orbitally $q$-close, so Th. 4.1.7 applies.

4.2. Sections over fine flag strata. We fix a parabolic subgroup $B \subset P_{0} \subset P$ with type $I_{0} \subset I$. We make the identification $X^{*}\left(\hat{E}_{P_{0}}\right)=X^{*}\left(L_{0}\right)$ as above. For a character $\lambda \in X^{*}\left(L_{0}\right)$, we denote by $\mathscr{L}_{P_{0}}(\lambda)$ (or $\mathscr{L}_{\mathcal{Z}, P_{0}}(\lambda)$ to avoid confusion) the attached line bundle on $G$-ZipFlag ${ }^{\left(\mathcal{Z}, P_{0}\right)} \simeq\left[\hat{E}_{P_{0}} \backslash G\right]$.

Remark 4.2.1. Since $\operatorname{Pic}(G)$ is finite, there exists an integer $m$ such that for all line bundle $\mathscr{L}$ on $G$-ZipFlag ${ }^{\left(\mathcal{Z}, P_{0}\right)}$, $\mathscr{L}^{m}=\mathscr{L}_{P_{0}}(\lambda)$ for some $\lambda \in X^{*}(L)$.

Definition 4.2.2. A stratification of a topological space $X$ is a decomposition $X=\bigsqcup_{i} X_{i}$ into locally closed subsets such that the closure of each $X_{i}$ is a union of $X_{j}$.

Definition 4.2.3 (Types of purity). A stratification of a scheme or stack $X$ is

(1) principally pure if for every stratum $S$, there exists a line bundle $\mathscr{L}$ on $X$ and a characteristic section

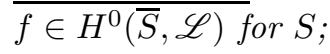


(2) uniformly principally pure if $\mathscr{L}$ may be chosen independently of $S$.

This definition applies to the stack $G$-ZipFlag ${ }^{\left(G, P_{0}\right)}$ endowed with the fine stratification. By Rmk. 4.2.1, we may equivalently ask for the existence of a character $\lambda \in X^{*}\left(L_{0}\right)$ such that $\mathscr{L}_{P_{0}}(\lambda)$ satisfies these conditions. Note that for $P_{0}=P$, this definition also applies to the stack $G$-Zip ${ }^{\mathcal{Z}}$, endowed with the zip stratification.

As we mentioned in the introduction, a key problem is to understand when the zip stratification of $G$-Zip ${ }^{\mathcal{Z}}$ is principally pure. If principal purity of the zip stratification would always hold (but it is not the case), that of the fine stratification of $G$-ZipFlag ${ }^{\left(\mathcal{Z}, P_{0}\right)}$ would follow by pulling back along the map $\Psi_{P_{0}}: G$-ZipFlag ${ }^{\left(\mathcal{Z}, P_{0}\right)} \rightarrow G$-Zip ${ }^{\mathcal{Z}_{0}}$.

We will give in $\$ 4.3$ an example where the zip stratification of $G$-Zip ${ }^{\mathcal{Z}}$ is not principally pure. We will prove below that when $\mathcal{Z}$ is maximal (in particular, if $\mathcal{Z}$ is of Hodge-type), then the zip stratification of $G$-Zip ${ }^{\mathcal{Z}}$ is uniformly principally pure. However, note that the zip datum $\mathcal{Z}_{0}$ is usually not maximal even if $\mathcal{Z}$ is.

As a consequence of Th. 4.1.7, we obtain Th. 3 of the introduction:

Theorem 4.2.4. Let $\left(\mathcal{Z}, P_{0}\right)$ be a flagged zip datum of exponent $n$ and write $q=p^{n}$. If there exists a $\mathcal{Z}_{0}$-ample, orbitally $q$-close character of $L_{0}$, then the fine stratification of $G$-ZipFlag $\left(\mathcal{Z}, P_{0}\right)$ is uniformly principally pure.

Proof. Apply Th. 4.1.7 to the stack $G$-Zip ${ }^{\mathcal{Z}_{0}}$ and pull back along $\Psi_{P_{0}}$.

Corollary 4.2.5. Let $G$ be a reductive group over $\mathbf{F}_{p}$. There exists $N \geq 1$ (depending only on $G$ ) such that for all $n \geq N$ and all flagged zip datum $\left(\mathcal{Z}, P_{0}\right)$ for $G$ of exponent $n$, the fine stratfication of $G$-ZipFlag ${ }^{\left(\mathcal{Z}, P_{0}\right)}$ is uniformly principally pure.

Another situation where Th. 4.2.4 applies is when we start with a cocharacter datum of characteristic 0. For large $p$, the reduction modulo $p$ will satisfy the conditions of the theorem. We will exploit this in $\$ 6$.

4.3. Counter-examples. In this section, we give a counter-example to principal purity. It is rather easy to find a counter-example to uniform principal purity. For example, consider a cocharacter zip datum $\left(G L_{4, \mathbf{F}_{p}}, \mu\right)$ where

$$
\mu: \mathbf{G}_{m, k} \rightarrow G L_{4, k}, \quad z \mapsto \operatorname{diag}\left(1, z, z^{2}, z^{3}\right) .
$$

Let $B \subset G L_{4, k}$ denote the Borel subgroup of upper-triangular matrices and $T \subset B$ the diagonal torus. The zip datum of exponent 1 attached to $\left(G L_{4, \mathbf{F}_{p}}, \mu\right)$ is $\mathcal{Z}:=\left(G L_{4, \mathbf{F}_{p}}, B, T, B_{-}, T, \varphi\right)$, where $B_{-}$is the opposite Borel. In this case, the zip strata of $G L_{4, k}$ coincide with the $B \times B_{-}$-orbits in $G L_{4, k}$. One can show that for $p=2$, there is no character $\lambda \in X^{*}(T)$ such that $\mathscr{V}(\chi)$ admits characteristic sections on all zip strata, so the zip stratification is not uniformly principally pure. However, it is clearly principally pure since it coincides with the Bruhat stratification.

To find a counter-example to principal purity, consider the following case. Consider the alternating matrix



Denote by $G:=S p(J)$ the symplectic group over $\mathbf{F}_{p}$ attached to $J$ and let $\mu$ be the cocharacter of $G$ defined by

$$
\mu: \mathbf{G}_{m, k} \rightarrow G_{k}, \quad z \mapsto \operatorname{diag}\left(z, z, 1,1, z^{-1}, z^{-1}\right) .
$$

Proposition 4.3.1. For $p=2$, the zip stratification of $G$-Zip ${ }^{\mu}$ is not principally pure.

First we let $p \geq 2$ be an arbitrary prime number. Let $T$ be the $\mathbf{F}_{p}$-split maximal torus given by the diagonal matrices in $G$ :

$$
T(R):=\left\{\operatorname{diag}\left(x_{1}, x_{2}, x_{3}, x_{3}^{-1}, x_{2}^{-1}, x_{1}^{-1}\right), \quad x_{1}, x_{2}, x_{3} \in R^{\times}\right\}
$$

for any $\mathbf{F}_{p}$-algebra $R$. A Borel subgroup $B$ over $\mathbf{F}_{p}$ containing $T$ is given by the lower-triangular matrices in $G$. For a triple $(a, b, c) \in \mathbf{Z}^{3}$, let $\lambda_{(a, b, c)} \in X^{*}(T)$ sending $\operatorname{diag}\left(x_{1}, x_{2}, x_{3}, x_{3}^{-1}, x_{2}^{-1}, x_{1}^{-1}\right)$ to $x_{1}^{a} x_{2}^{b} x_{3}^{c}$. Identify $X^{*}(T)$ with $\mathbf{Z}^{3}$ via this isomorphism. Denoting by $\left(e_{1}, e_{2}, e_{3}\right)$ the standard basis of $\mathbf{Z}^{3}$, the $T$-roots of $G$, the positive roots, the simple roots are respectively:

$$
\begin{aligned}
\Phi & :=\left\{e_{i} \pm e_{j}, 1 \leq i \neq j \leq 3\right\} \cup\left\{ \pm 2 e_{i}, 1 \leq i \leq 3\right\} \\
\Phi^{+} & :=\left\{e_{i} \pm e_{j}, 1 \leq i<j \leq 3\right\} \cup\left\{2 e_{i}, 1 \leq i \leq 3\right\} \\
\Delta & :=\left\{\alpha_{1}, \alpha_{2}, \alpha_{3}\right\}
\end{aligned}
$$

where $\alpha_{1}, \alpha_{2}, \alpha_{3}$ are defined by

$$
\alpha_{1}:=e_{1}-e_{2} \quad ; \quad \alpha_{2}:=e_{2}-e_{3} \quad ; \quad \alpha_{3}:=2 e_{3} .
$$


The Weyl group $W:=N_{G}(T) / T$ is the group of permutations $\sigma \in \mathfrak{S}_{6}$ satisfying $\sigma(i)+\sigma(7-i)=7$ for all $1 \leq i \leq 6$. Following [EvdG09, we denote a permutation $\sigma \in W$ by $[\sigma(1) \sigma(2) \sigma(3)]$ (these three values determine $\sigma \in W$ uniquely). The identity element $e \in W$ is [123] and the simple reflections are

$$
s_{\alpha_{1}}=[213] \quad ; \quad s_{\alpha_{2}}=[132] \quad ; \quad s_{\alpha_{3}}=[124] .
$$

The longest element in $W$ is $w_{0}=[654]$ and has length 9 .

Define a maximal parabolic $P$ containing $B$ such that for any $\mathbf{F}_{p}$-algebra $R$, the group $P(R)$ is the set of elements of $G(R)$ of the form:

$$
\left(\begin{array}{ccc}
A & 0 & 0 \\
* & B & 0 \\
* & * & C
\end{array}\right), \quad A, B, C \in G L_{2}(R)
$$

One sees immediately that such a matrix satisfies the conditions $B \in S L_{2}(R)$ and $C=J_{0}{ }^{t} A^{-1} J_{0}$ with $J_{0}:=\left(\begin{array}{ll}0 & 1 \\ 1 & 0\end{array}\right)$. In particular, the Levi subgroup $L$ of $P$ containing $T$ is isomorphic to $G L_{2, \mathbf{F}_{p}} \times S L_{2, \mathbf{F}_{p}}$ and $W_{L}$ identifies with the subgroup $W_{L}=\left\langle s_{\alpha_{1}}, s_{\alpha_{3}}\right\rangle$. The longest element of $W_{L}$ is $w_{0, L}:=$ [214] (length 2). We also define:

$$
z:=w_{0} w_{0, L}=[563] \text {. }
$$

Let $Q$ be the opposite parabolic subgroup of $P$ with respect to $L$. We obtain a zip datum $\mathcal{Z}:=(G, P, L, Q, L, \varphi)$.

Here is a representation of the zip strata. For $w, w^{\prime} \in W$, an arrow $w \rightarrow w^{\prime}$ indicates the closure relation $G_{w} \subset \bar{G}_{w^{\prime}}$. In this example, the order $\preceq$ coincides with the restriction of the Bruhat order $\leq$ to ${ }^{I} W$.

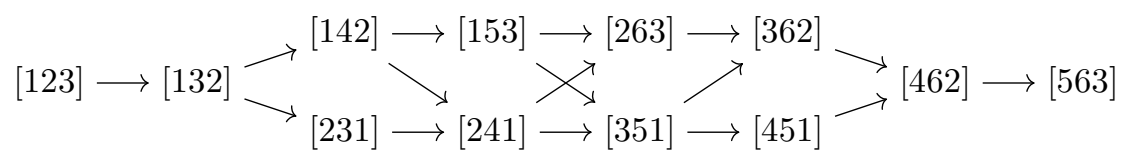

In the following, we take $\chi:=\lambda_{(1,0,0)}$, which is the fundamental weight of $Q$. Consider the element

$$
w:=[351] \in{ }^{I} W .
$$

One has $\ell(w)=4$. The set $E_{w}$ of "lower neighbors" of $w$ in $W$ consists of 4 elements:

$$
\begin{aligned}
& w_{1}:=[153]=w s_{\alpha_{1}} \quad \text { with } \quad \alpha_{1}=e_{1}-e_{3} \\
& w_{2}:=[241]=w s_{\alpha_{2}} \quad \text { with } \quad \alpha_{2}=e_{1}+e_{2} \\
& w_{3}:=[315]=w s_{\alpha_{3}} \quad \text { with } \quad \alpha_{3}=e_{2}-e_{3} \\
& w_{4}:=[321]=w s_{\alpha_{4}} \quad \text { with } \quad \alpha_{4}=2 e_{2}
\end{aligned}
$$

An easy computation shows that the multiplicities $n_{\alpha}$ as in (4.1.1) are given by:

$$
\begin{aligned}
& n_{\alpha_{1}}=p-1 \\
& n_{\alpha_{2}}=2 p-1 \\
& n_{\alpha_{3}}=p-2 \\
& n_{\alpha_{2}}=p-1
\end{aligned}
$$

Hence for $p=2$, one has $n_{\alpha_{3}}=0$, so $g_{w}$ is non-vanishing along $X_{w_{3}}$. This shows that no power of $h_{w, \chi}$ extends to a characteristic function of $G_{w}$. Actually, one can show that no power of $h_{w, \chi}$ extends at all to $\bar{G}_{w}$.

\section{FunCtORIALity AND DisCRETE FIBER THEOREM}

5.1. Discrete fiber theorem. We consider a morphism $f: \mathcal{Z}_{1} \rightarrow \mathcal{Z}_{2}$ of zip data of exponent $n$, with $\mathcal{Z}_{i}=$ $\left(G_{i}, P_{i}, L_{i}, Q_{i}, M_{i}, \varphi^{n}\right)$, and let $I_{i}$ be the type of $P_{i}$ (for $\left.i=1,2\right)$. We say that $f$ has central kernel if the underlying group homomorphism $f: G_{1} \rightarrow G_{2}$ has central (scheme-theoretic) kernel.

Theorem 5.1.1. Let $f: \mathcal{Z}_{1} \rightarrow \mathcal{Z}_{2}$ be a morphism with central kernel. Then the attached morphism of stacks

$$
\tilde{f}: G_{1} \text { Zip }^{\mathcal{Z}_{1}} \rightarrow G_{2} \text {-Zip }^{\mathcal{Z}_{2}}
$$

has discrete fibers on the underlying topological spaces.

Proof. We first reduce the proof to the case when $f$ is a closed embedding. Set $K:=\operatorname{Ker}(f)$ and define $\tilde{\square}=\square / K$ for $\square=G_{1}, P_{1}, L_{1}, Q_{1}, M_{1}$. We get a zip datum $\tilde{\mathcal{Z}}_{1}:=\left(\tilde{G}_{1}, \tilde{P}_{1}, \tilde{L}_{1}, \tilde{Q}_{1}, \tilde{M}_{1}, \varphi^{n}\right)$. The natural projection $\pi: G_{1} \rightarrow \tilde{G}_{1}$ induces a map of zip data $\mathcal{Z}_{1} \rightarrow \tilde{\mathcal{Z}}_{1}$ and the induced map

$$
\tilde{\pi}: G_{1}-Z_{i p} \mathcal{Z}^{\mathcal{Z}_{1}} \rightarrow \tilde{G}_{1} \text {-Zip } \tilde{\mathcal{Z}}^{\tilde{\mathcal{B}}_{1}}
$$


is a bijection on the underlying topological spaces, by the proof of [KW] Prop. 3.20]. We may factor the map $f: G_{1} \rightarrow G_{2}$ as the composition $G_{1} \stackrel{\pi}{\rightarrow} \tilde{G}_{1} \stackrel{\iota}{\rightarrow} G_{2}$ where $\iota$ is a closed embedding, so it suffices to prove the result for the map $\iota$. Hence we assume from now on that $f$ is a closed embedding.

Lemma 5.1.2. Let $\chi_{2} \in X^{*}\left(L_{2}\right)$ be a $\mathcal{Z}_{2}$-ample character. Then the restriction $\chi_{1}=\left.\chi_{2}\right|_{L_{1}} \in X^{*}\left(L_{1}\right)$ is $\mathcal{Z}_{1}$-ample.

Proof of Lemma 5.1.2. We first show that $f^{-1}\left(Q_{2}\right)=Q_{1}$. By definition of a map of zip data, one has $f\left(Q_{1}\right) \subset Q_{2}$, so $Q_{1} \subset f^{-1}\left(Q_{2}\right)$. In particular, $f^{-1}\left(Q_{2}\right)$ is a parabolic subgroup of $G_{1}$. Then $f^{-1}\left(V_{2}\right)$ is a unipotent normal subgroup of $f^{-1}\left(Q_{2}\right)$, hence its identity component is contained in the unipotent radical of $f^{-1}\left(Q_{2}\right)$. Again by definition we have $V_{1} \subset f^{-1}\left(V_{2}\right)$, so we deduce that $V_{1}$ is contained in the unipotent radical of $f^{-1}\left(Q_{2}\right)$. This implies $Q_{1}=f^{-1}\left(Q_{2}\right)$. Since $f$ is defined over $\mathbf{F}_{p}$, we obtain an embedding $G_{1} / \varphi^{-n}\left(Q_{1}\right) \rightarrow G_{2} / \varphi^{-n}\left(Q_{2}\right)$. This proves the result.

We first prove Th. 5.1.1 for large $n$. More precisely, one has the following lemma:

Lemma 5.1.3. Assume that there exists a $\mathcal{Z}_{2}$-ample character $\chi_{2} \in X^{*}\left(L_{2}\right)$ such that $\chi_{1}:=\left.\chi_{2}\right|_{L_{1}}$ is $p^{n}$-small. Then $\tilde{f}$ has discrete fibers.

Proof of Lemma 5.1.3. By Lem. 55.1.2 above, $\chi_{1}$ is $\mathcal{Z}_{1}$-ample, so we may apply Th. 4.1.7 to $\chi_{1}$. If $\tilde{f}$ doesn't have discrete fibers, then we can find two zip strata $C_{1} \subset G_{1}$ and $C_{1}^{\prime} \subset \overline{C_{1}}$ mapping to the same zip stratum $C_{2}$ of $G_{2}$. By Th. 4.1.7, there exists $N \geq 1$ such that $\mathscr{V}\left(N \chi_{1}\right)$ admits a section $H_{1}$ on $\overline{C_{1}}$ with non-vanishing locus $C_{1}$. We can also find $r \geq 1$ and a non-vanishing section $H_{2}$ of $\mathscr{V}\left(r \chi_{2}\right)$ on $C_{2}$. The pull-back of $H_{2}^{N}$ to $C_{1}$ agrees (up to a nonzero scalar) with $H_{1}^{r}$, since they are both nonzero sections of $\mathscr{V}\left(N r \chi_{1}\right)$, and the space $H^{0}\left(C_{1}, \mathscr{V}\left(N r \chi_{1}\right)\right.$ has dimension one by [KW, Prop.1.18]. This contradicts the fact that $H_{1}$ vanishes on $C_{1}^{\prime}$.

We now prove the general case. Fix an integer $A$ such that all the groups $P_{i}, L_{i}, Q_{i}, M_{i}$ for $i=1,2$ are defined over $\mathbf{F}_{p^{A}}$. For an integer $N \equiv 1(\bmod A)$, we consider new zip data

$$
\begin{aligned}
& \mathcal{Z}_{1, N}=\left(G_{1}, P_{1}, L_{1}, Q_{1}, M_{1}, \varphi^{n N}\right) \\
& \mathcal{Z}_{2, N}=\left(G_{2}, P_{2}, L_{2}, Q_{2}, M_{2}, \varphi^{n N}\right) .
\end{aligned}
$$

By our assumption on $N$ and $A$, the tuples $\mathcal{Z}_{1, N}$ and $\mathcal{Z}_{2, N}$ are zip data of exponent $N n$. The map $f$ induces a morphism of zip data $f_{N}: \mathcal{Z}_{1, N} \rightarrow \mathcal{Z}_{2, N}$ and a morphism of stacks

$$
\tilde{f}_{N}: G_{1}-\text { Zip }^{\mathcal{Z}_{1, N}} \rightarrow G_{2} \text {-Zip }^{\mathcal{Z}_{2, N}}
$$

By the parametrization (1.6.1), there exists a finite subset $\Sigma_{1} \subset G_{1}(k)$ (resp. $\Sigma_{2} \subset G_{2}(k)$ ), independent of $N$, which is a system of representatives of the $\mathcal{Z}_{1, N}$-strata in $G_{1}$ (resp. the $\mathcal{Z}_{2, N}$-strata in $G_{2}$ ) for all $N$. Hence $f_{N}$ induces a map of sets

$$
\Psi_{N}: \Sigma_{1} \rightarrow \Sigma_{2}
$$

We believe that $\Psi_{N}$ is independent of $N$, but we only need the following weaker result:

Lemma 5.1.4. Fix an integer $C \geq 1$. Assume that $x, y \in \Sigma_{1}$ satisfy $\Psi_{1}(x)=\Psi_{1}(y)$. Then there exists arbitrary large $N \equiv 1(\bmod C)$ such that $\Psi_{N}(x)=\Psi_{N}(y)$.

Proof of Lemma 5.1.4. Since $\Psi_{1}(x)=\Psi_{1}(y)$, the elements $f(x)$ and $f(y)$ are in the same $\mathcal{Z}_{2}$-zip stratum. Hence we can find $u \in U_{2}, v \in V_{2}$ and $a \in L_{2}$ such that

$$
f(y)=u a f(x) \varphi^{n}(a)^{-1} v
$$

Choose $B \geq 1$ such that $a \in G\left(\mathbf{F}_{p^{B}}\right)$. Then for $N \equiv 1(\bmod A B C)$, we can write $f(y)=u a f(x) \varphi^{n N}(a)^{-1} v$, which shows that $\Psi_{N}(x)=\Psi_{N}(y)$.

To finish the proof of Th. 5.1.1, denote by $\preceq_{N}$ the relation (3.3.2) for the zip datum $\mathcal{Z}_{1, N}$. Assume that $\tilde{f}=\tilde{f}_{1}$ doesn't have discrete fibers. Then there exists $x, y \in \Sigma_{1}$ such that $x \preceq_{1} y$ and $\Psi_{1}(x)=\Psi_{1}(y)$. By Prop. 3.3.2, there exists $h \in L_{1}$ such that $h x \varphi^{n}(x)^{-1} \leq y$. Choose $C \geq 1$ such that $h \in G\left(\mathbf{F}_{p^{C}}\right)$. Then

$$
h x \varphi^{n N}(x)^{-1}=h x \varphi^{n}(x)^{-1} \leq y
$$

for all $N \equiv 1(\bmod C)$, which shows that $x \preceq_{N} y$. By Lem. 5.1.4 there exists arbitrary large $N \equiv 1(\bmod C)$ such that $\Psi_{N}(x)=\Psi_{N}(y)$, hence the map $\tilde{f_{N}}$ does not have discrete fibers for each such $N$. But for large $N$, we can find a $\mathcal{Z}_{2}$-ample character of $L_{2}$ restricting to a $p^{N n}$-small character of $L_{1}$. By Lem. 5.1.3 the map $\tilde{f}_{N}$ has discrete fibers, hence a contradiction.

Corollary 5.1.5. Let $f: \mathcal{Z}_{1} \rightarrow \mathcal{Z}_{2}$ be a morphism with central kernel. If the zip stratification of $\mathcal{Z}_{2}$ is principally pure (resp. uniformly principally pure), then so is the zip stratification of $\mathcal{Z}_{1}$. 
Example 5.1.6. We expand on the counter-example in \$4.3. Take $G=S p(J)$ as in 4.3.2) and $\mu: \mathbf{G}_{m, k} \rightarrow G_{k}$ as in 4.3.3). There is a natural embedding $\iota: G \rightarrow G L_{6, \mathbf{F}_{p}}$, and the cocharacter datum $\left(G L_{6, \mathbf{F}_{P}}, \iota \circ \mu\right)$ yields a zip datum $\mathcal{Z}^{\prime}$ for $G L_{6, \mathbf{F}_{p}}$. For $p=2$, Cor. 5.1.5 implies that the zip stratification of $G L_{6}$-Zip ${ }^{\mathcal{Z}^{\prime}}$ is not principally pure.

\subsection{Corollaries of the discrete-fiber theorem.}

Corollary 5.2.1. Let $\mathcal{Z}=\left(G, P, L, Q, M, \varphi^{n}\right)$ be a zip datum and set $q:=p^{n}$. Assume $G$ admits an embedding $G \rightarrow G L_{N, \mathbf{F}_{p}}$ defined over $\mathbf{F}_{p}$. If $q \geq N$, then the zip stratification of $G$ is uniformly principally pure.

Proof. By Cor. 5.1.5, it suffices to consider the case $G=G L_{N, \mathbf{F}_{p}}$. We may assume that $L$ is the Levi subgroup corresponding to a decomposition $N=N_{1}+\ldots+N_{r}$, given by diagonal blocks of size $N_{1}, \ldots, N_{r}$. We identify a character of $L$ with a tuple $\left(a_{1}, \ldots, a_{r}\right) \in \mathbf{Z}^{r}$. Consider the character $\lambda=(r, r-1, \ldots, 1)$. This character is $\mathcal{Z}$-ample and orbitally $q$-close for $q \geq r$, hence the result.

The shortcoming of Th. 4.1.7 is that the set of $\mathcal{Z}$-ample, orbitally $q$-close characters could be empty for small values of $p$. However, we have the following result:

Corollary 5.2.2. Let $(G, \mu)$ be a maximal cocharacter datum over $\mathbf{F}_{p}$, and let $\chi$ be a maximal character of $L$ (Def. 1.4.3). Then there exists $N \geq 1$ such that the line bundle $\mathscr{V}(N \chi)$ admits characteristic sections on all zip strata of $G$. In particular, the zip stratification of $G$-Zip ${ }^{\mu}$ is uniformly principally pure.

Proof. This follows immediately from Cor. 5.1.5 and Cor. 4.1.9.

Corollary 5.2.3. Let $(G, \mu)$ be a cocharacter datum over $\mathbf{F}_{p}$ and assume $\mu$ is minuscule. For $p>2$, the zip stratification of $G$-Zip ${ }^{\mu}$ is uniformly principally pure.

Proof. Consider the adjoint representation Ad $: G \rightarrow G L(\mathfrak{g})$ where $\mathfrak{g}$ is the Lie algebra of $G$. Since $\mu$ is minuscule, the cocharacter Ad $\circ \mu: \mathbf{G}_{m, k} \rightarrow G L(\mathfrak{g})_{k}$ has only weights $-1,0,1$. Hence we reduce to the case of $G L(V)$ (for a finite-dimensional $\mathbf{F}_{p}$-vector space $V$ ) and a zip datum whose attached parabolic $P$ is the stabilizer of a flag $0 \subset V_{1} \subset V_{2} \subset V$. It is clear that $P$ admits an $\mathcal{Z}$-ample orbitally 3 -close character, which proves the result.

5.3. Functoriality of zip flags. Consider two zip data $\mathcal{Z}=\left(G, P, L, Q, M, \varphi^{n}\right)$ and $\mathcal{Z}^{\prime}=\left(G^{\prime}, P^{\prime}, L^{\prime}, Q^{\prime}, M^{\prime}, \varphi^{n}\right)$ of exponent $n$. Choose parabolic subgroups $P_{0} \subset P$ and $P_{0}^{\prime} \subset P^{\prime}$, together with Levi subgroups $L_{0} \subset P_{0}$ and $L_{0}^{\prime} \subset P_{0}^{\prime}$ satisfying $L_{0} \subset L$ and $L_{0}^{\prime} \subset L^{\prime}$. Denote by $U_{0}$ and $U_{0}^{\prime}$ the unipotent radicals of $P_{0}$ and $P_{0}^{\prime}$ respectively.

Let $f: \mathcal{Z} \rightarrow \mathcal{Z}^{\prime}$ be a morphism of zip data satisfying the additional conditions:

(i) $f\left(L_{0}\right) \subset L_{0}^{\prime}$

(ii) $f\left(U_{0}\right) \subset U_{0}^{\prime}$.

For example, these conditions are satisfied if $\left(P_{0}, L_{0}\right)$ and $\left(P_{0}^{\prime}, L_{0}^{\prime}\right)$ are attached to cocharacters $\mu_{0}: \mathbf{G}_{m, k} \rightarrow G_{k}$ and $\mu_{0}^{\prime}=f \circ \mu_{0}$ respectively (\$1.2). The map $f$ sends the subgroup $E_{\mathcal{Z}} \subset G_{k} \times G_{k}$ to $E_{\mathcal{Z}^{\prime}} \subset G_{k}^{\prime} \times G_{k}^{\prime}$. Since $f\left(P_{0}\right) \subset P_{0}^{\prime}$, we also have $f\left(\hat{E}_{P_{0}}^{\mathcal{Z}}\right) \subset \hat{E}_{P_{0}^{\prime}}^{\mathcal{Z}^{\prime}}$. Furthermore, define zip data $\mathcal{Z}_{0}:=\left(G, P_{0}, L_{0}, Q_{0}, M_{0}, \varphi^{n}\right)$ and $\mathcal{Z}_{0}^{\prime}:=$ $\left(G^{\prime}, P_{0}^{\prime}, L_{0}^{\prime}, Q_{0}^{\prime}, M_{0}^{\prime}, \varphi^{n}\right)$ as in (3.1.1). It follows from our assumptions that $f$ induces a morphism of zip data $\mathcal{Z}_{0} \rightarrow \mathcal{Z}_{0}^{\prime}$ and a commutative diagram:

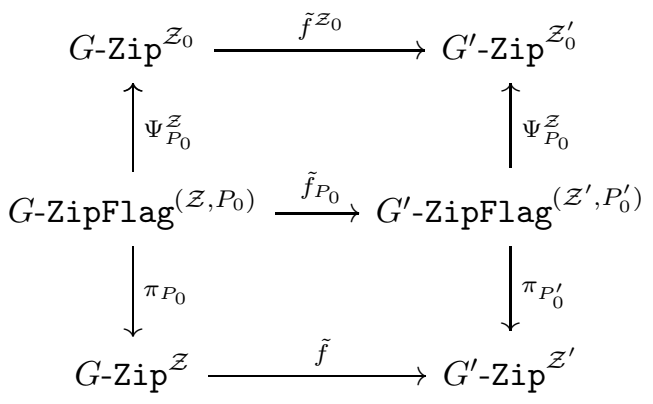

Since the diagram commutes, the map $\tilde{f}_{P_{0}}$ induces a map between the fibers of $\pi_{P_{0}}$ and $\pi_{P_{0}^{\prime}}$. These fibers can be identified with $P / P_{0}$ and $P^{\prime} / P_{0}^{\prime}$ respectively, and the map $\tilde{f}_{P_{0}}$ then identifies with the natural map $P / P_{0} \rightarrow P^{\prime} / P_{0}^{\prime}$ induced by $f$.

\section{FlaG SPACES AND STRATiFiCATiOns}

6.1. Shimura varieties of Hodge-type. Let $(\mathbf{G}, X)$ be a Shimura datum of Hodge type with reflex field $E \subset \mathbf{C}$, given by a reductive group $\mathbf{G}$ over $\mathbf{Q}$ and a $\mathbf{G}(\mathbf{R})$-conjugacy class of morphisms $\mathbf{S} \rightarrow \mathbf{G}_{\mathbf{R}}$. For $h_{0} \in X$, define $\mu_{0}: \mathbf{G}_{m, \mathbf{C}} \rightarrow \mathbf{G}_{\mathbf{C}}$ as $\mu_{0}(z)=h_{0, \mathbf{C}}(z, 1)$ using the identification $\mathbf{S}_{\mathbf{C}} \simeq \mathbf{G}_{m, \mathbf{C}} \times \mathbf{G}_{m, \mathbf{C}}$ given by $z \mapsto(z, \bar{z})$ on $\mathbf{R}$-points.

We choose an integer $m \geq 1$ and a sufficiently small compact open subgroup $K \subset \mathbf{G}\left(\mathbf{A}_{f}\right)$ such that the following properties hold: 
(1) There is a reductive model $\mathcal{G}$ of $\mathbf{G}$ over $\mathbf{Z}\left[\frac{1}{m}\right]$.

(2) The Shimura variety $\mathbb{S}_{K}:=S h_{K}(\mathbf{G}, X)$ admits a smooth integral model $\mathcal{S}_{K}$ over $R:=\mathcal{O}_{E}\left[\frac{1}{m}\right]$. This model can be obtained by glueing the $\mathcal{O}_{E, \mathfrak{p}}$-models of [Vas99] and [Kis10] for primes $\mathfrak{p}$ of good reduction.

(3) There is a cocharacter $\mu$ of $\mathcal{G}_{R}$ such that $\mu_{\mathbf{C}}$ lies in the conjugacy class of $\mu_{0}$.

For each prime $\mathfrak{p}$ in $R$ lying above a prime number $p$, we obtain a cocharacter datum $(G, \mu)$ where $G:=\mathbf{G} \otimes \mathbf{F}_{p}$.

Let $f: \mathscr{A} \rightarrow \mathcal{S}_{K}$ be the universal abelian scheme obtained by pull-back from the Siegel case. For a prime $\mathfrak{p}$ of $R$, define $S_{K}:=\mathcal{S}_{K} \otimes R / \mathfrak{p}$ the special fiber of $\mathcal{S}_{K}$ and $A:=\mathscr{A} \otimes R / \mathfrak{p}$. The de Rham cohomology $H_{d R}^{1}\left(A / S_{K}\right)$ together with its Hodge filtration and conjugate filtration naturally defines a $G$-zip over $S_{K}$ ([Zha, Th. 2.4.1]). This induces a smooth morphism of stacks ([Zha, Th. 3.1.2])

$$
\zeta: S_{K} \longrightarrow G \text {-Zip }{ }^{\mu}
$$

The geometric fibers of $\zeta$ are the Ekedahl-Oort strata of $S_{K}$. See the introduction of GKb for further references and remarks about $\zeta$. The Hodge line bundle of $\mathcal{S}_{K}$ is defined as

$$
\omega:=f_{*}\left(\operatorname{det}\left(\Omega_{\mathscr{A} / \mathcal{S}_{K}}\right)\right) \text {. }
$$

It is ample on $\mathcal{S}_{K}$. Denote by $\mathbf{P} \subset \mathbf{G}_{\overline{\mathbf{Q}}}$ the parabolic subgroup attached to $\left(\mathbf{G}, \mu_{E}\right)$. For a character $\lambda \in X^{*}(\mathbf{P})$, there is an automorphic line bundle $\mathcal{V}_{K}(\lambda)$ on $\mathcal{S}_{K}$ attached to $\lambda$ and its special fiber coincides with the line bundle $\zeta^{*}(\mathcal{V}(\lambda))$. Furthermore, there exists a character $\eta_{\omega} \in X^{*}(\mathbf{P})$ such that $\omega=\mathcal{V}\left(\eta_{\omega}\right)$.

6.2. Flag spaces. Choose a parabolic subgroup $\mathbf{P}_{0} \subset \mathbf{P}$. We can find a finite extension $E \subset E_{0} \subset \mathbf{C}$ such that $\mathbf{P}$ and $\mathbf{P}_{0}$ admit models $\mathcal{P}$ and $\mathcal{P}_{0}$ over $R_{0}:=\mathcal{O}_{E_{0}}\left[\frac{1}{m}\right]$ and such that all automorphic line bundles are defined over $R_{0}$.

The scheme $\mathcal{S}_{K}$ carries a universal $\mathcal{P}$-torsor $\mathfrak{P}$ arising from the Hodge filtration of the de Rham cohomology of $\mathscr{A}$, as defined in [Mad, Prop. 5.3.4] (see also $\S 2.1 .5$ in [GKb]). Then the quotient of $\mathfrak{P}$ by the group $\mathcal{P}_{0}$ defines a smooth $\mathcal{P} / \mathcal{P}_{0}$-bundle

$$
\pi: \mathcal{F} l_{K} \longrightarrow \mathcal{S}_{K} \otimes R_{0}
$$

It generalizes the flag space considered by Ekedahl and Van der Geer in EvdG09 and by the authors in GKb. We call $\mathcal{F} l_{K}$ the flag space attached to the parabolic $\mathbf{P}_{0}$. Define $\mathbb{F} l_{K}:=\mathcal{F} l_{K} \otimes E_{0}$. For a given prime $\mathfrak{p}_{0} \subset R_{0}$ lying above $\mathfrak{p} \subset R$ and $p \in \mathbf{Z}$, write $F l_{K}$ for the reduction of $\mathcal{F} l_{K}$ modulo $\mathfrak{p}_{0}$. As explained in GKb] (10.3.3), we have a Cartesian diagram:

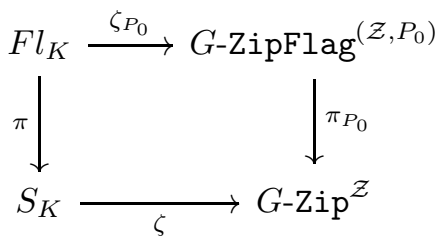

A character $\lambda \in X^{*}\left(\mathbf{P}_{0}\right)$ naturally gives rise to a line bundle $\mathcal{L}_{K}(\lambda)$ on $\mathcal{F} l_{K}$ (see [Jan03] §5.8). On the special fiber, one has the following formula:

$$
\mathscr{L}_{K}(\lambda)=\zeta_{P_{0}}^{*}\left(\mathscr{L}_{P_{0}}(\lambda)\right)
$$

6.3. Stratifications. We define a stratification on $F l_{K}$ by pulling back fine flag strata of $G$-ZipFlag ${ }^{\left(\mathcal{Z}, P_{0}\right)}$ along the smooth morphism $\zeta_{P_{0}}$. Equivalently, we define strata as the fibers of the composition

$$
F l_{K} \stackrel{\zeta_{P_{0}}}{\longrightarrow} G \text {-ZipFlag }\left(\mathcal{Z}, P_{0}\right) \stackrel{\Psi_{P_{0}}}{\longrightarrow} G \text {-Zip } \mathcal{Z}^{\mathcal{Z}_{0}}
$$

For $w \in{ }^{I_{0}} W \cup W^{J_{0}}$, define the corresponding fine flag strata as:

$$
F l_{w}:=\zeta_{P_{0}}^{-1}\left(Z_{P_{0}, w}\right)
$$

endowed with the reduced structure. Similarly, for $w \in{ }^{I} W \cup W^{J}$, define Ekedahl-Oort strata of $S_{K}$ by:

$$
S_{w}:=\zeta^{-1}\left(Z_{P, w}\right) .
$$

For $w \in{ }^{I_{0}} W^{J_{0}}$, define the coarse flag strata by

$$
\mathbf{F}_{w}:=\zeta_{P_{0}}^{-1}\left(\mathbf{Z}_{P_{0}, w}\right) .
$$

The following is a simple consequence of the results in sections 213

\section{Proposition 6.3.1.}

(1) For $w \in{ }^{I_{0}} W \cup W^{J_{0}}$, the fine flag stratum $F l_{w}$ is smooth of dimension $\ell(w)$.

(2) For $w \in{ }^{I_{0}} W^{J_{0}}$, the coarse flag stratum $\mathbf{F}_{w}$ is smooth of dimension $\ell(w)+\ell\left(w_{0, J_{0}}\right)-\ell\left(w_{0, I_{w}}\right)-\operatorname{dim}\left(P_{0}\right)$, where $I_{w}:=J_{0} \cap w^{-1} I_{0} w$.

(3) The Zariski closure of a fine (resp. coarse) stratum is a union of fine (resp. coarse) strata. 
(4) The image by $\pi_{P_{0}}^{*}$ of a fine stratum is a union of Ekedahl-Oort strata.

(5) For $w \in{ }^{I_{0}} W \cup W^{J_{0}}$ one has $\pi_{P_{0}}^{*}\left(F l_{w}\right)=S_{w}$ and the map $\pi: F l_{w} \rightarrow S_{w}$ is finite.

We now prove Th. 3 of the introduction, which states that for large $p$, the fine strata $F l_{w}$ of the flag space $F l_{K}$ are affine and define a uniformly principally pure stratification for large $p$, which generalizes [EvdG09, Prop. 10.5(ii)] in the Siegel case.

Theorem 6.3.2. There exists an integer $N$ depending on $\mathbf{G}, X$ and $K$ such that for all $p \geq N$, the Shimura variety $S h_{K}(\mathbf{G}, X)$ has good reduction at all places $\mathfrak{p} \mid p$ and the following holds:

(1) The fine stratification of $F l_{K}$ is uniformly principally pure.

(2) If the closure $\bar{S}$ of a fine flag stratum $S \subset F l_{K}$ is proper, then $S$ is affine.

Proof. Property (1) is a simple consequence of Th. 4.2.4 so it remains to prove (2). Choose an $\mathcal{Z}_{0}$-ample character $\eta \in X^{*}\left(\mathbf{P}_{0}\right)$. By Rmk. 4.1.5 the line bundle $\mathscr{L}(\eta)$ is relatively ample with respect to the morphism $\pi: \mathcal{F} l_{K} \rightarrow \mathcal{S}_{K}$. By standard arguments, there exists an integer $m \geq 1$ such that the line bundle

$$
\mathcal{R}:=\mathscr{L}_{K}(\eta) \otimes\left(\pi^{*} \omega\right)^{m}=\mathscr{L}_{K}\left(\eta+m \eta_{\omega}\right)
$$

is ample on $\mathcal{F} l_{K}$. The character $\eta+m \eta_{\omega}$ is $\mathcal{Z}_{0}$-ample and for large prime numbers $p$, it is also $p$-small. Hence there exists an integer $N$ such that for all primes $p \geq N$, the ample line bundle $\mathcal{R}$ admits characteristic sections on all fine flag strata, again by Th. 4.2.4. This concludes the proof.

6.4. Is there an analogue of $G$-Zip ${ }^{\mathcal{Z}_{0}}$ at the level of Shimura varieties? We have seen that the flag spaces $F l_{K}$ are counterparts of the stacks $G$-ZipFlag ${ }^{\left(\mathcal{Z}, P_{0}\right)}$ in the context of Shimura varieties. Likewise, it would be interesting to construct a counterpart $S_{P_{0}}^{?}$ to the stack $G$-Zip ${ }^{\mathcal{Z}_{0}}$. Note that $\mathcal{Z}_{0}$ is usually not a zip datum arising from a cocharacter datum. The properties that should be satisfied by the scheme $S_{P_{0}}^{\text {? }}$ are the following: There should exist natural morphisms $\Psi_{P_{0}}^{?}: F l_{K} \rightarrow S_{K}^{?}$ and $\zeta^{?}: S_{P_{0}}^{?} \rightarrow G$-Zip ${ }^{\mathcal{Z}_{0}}$ (i.e $S_{P_{0}}^{?}$ should carry a universal $G$-zip of type $\mathcal{Z}_{0}$ ). These maps should give rise to a commutative diagram

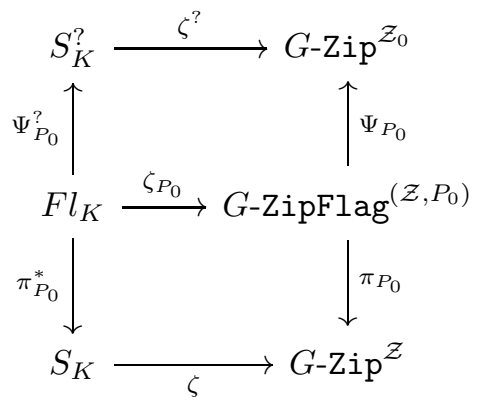

such that both squares are Cartesian, that is

$$
F l_{K} \simeq S_{K}^{?} \times{ }_{G-\text { Zip }_{0} z_{0}} G \text {-ZipFlag }{ }^{\left(\mathcal{Z}, P_{0}\right)} .
$$

In particular, the map $\Psi_{P_{0}}^{?}$ should be an affine bundle with fibers isomorphic to $\mathbf{A}^{r}$ where $r=\operatorname{dim}\left(G / P_{0}\right)$.

As mentioned in the introduction, one of the main aspects of our program initiated in [GKb] is the analogy/connection between Griffiths-Schmid manifolds and stacks of $G$-Zips, see esp. $\$ 1.4$ of loc. cit. More precisely, it appears that stacks of $G$-Zips may be viewed as characteristic $p$ analogues of Mumford-Tate domains. Recall that Mumford-Tate domains are the Hodge-theoretic generalizations of period domains which admit Griffiths-Schmid manifolds as arithmetic quotients.

This analogy may shed some light on the problem of constructing a pair $\left(S_{K}^{?}, \Psi_{P_{0}}^{?}\right)$ as above. Specifically, in GGK13, Green-Griffiths-Kerr introduce and study several complex manifolds associated with a Mumford-Tate domain $D$. In the terminology and notation of loc. cit. (see esp. Chap. 6), these include the correspondence space $\mathcal{W}$, the incidence variety $\mathcal{J}$ and the cycle space $\mathcal{U}$. For more on the cycle space, see [FHW06].

The "basic diagram" of [GGK13, Chap. 6] is:




It is reminiscent of the desired diagram above for the hypothetical pair $\left(S_{K}^{?}, \Psi_{P_{0}}^{?}\right)$. For example, it is suggestive that, as explained in loc. cit., the maps $\mathcal{W} \rightarrow D$ and $\mathcal{W} \rightarrow \mathcal{J}$ are contractible. This should be compared with the above desideratum that $\Psi_{P_{0}}^{?}$ be an affine bundle.

\section{REFERENCES}

[ABD $\left.{ }^{+66}\right]$ M. Artin, J. E. Bertin, M. Demazure, P. Gabriel, A. Grothendieck, M. Raynaud, and J.-P. Serre, SGA3: Schémas en groupes., vol. 1963/64, Institut des Hautes Études Scientifiques, Paris, 1965/1966.

[BGKS] Y. Brunebarbe, W. Goldring, J.-S. Koskivirta, and B. Stroh, Ampleness of automorphic bundles on zip-schemes, In preparation.

[Del79] P. Deligne, Variétés de Shimura: Interprétation modulaire, et techniques de construction de modèles canoniques, Automorphic Forms, representations, and L-Functions (July 11 - August 5, 1977, Corvallis, OR, USA) (A. Borel and W. Casselman, eds.), Proc. Symp. Pure Math., vol. 33, Amer. Math. Soc., Providence, RI, USA, 1979, pp. 247-289.

[EvdG09] T. Ekedahl and G. van der Geer, Cycle classes of the E-O stratification on the moduli of abelian varieties, Algebra, arithmetic and geometry (June 2009, Penn. State U., PA, USA) (Y. Tschinkel and Y. Zarhin, eds.), Progress in Math., vol. 269, Birkhäuser, Boston, MA, USA, 2009, pp. 567-636.

[FHW06] G. Fels, A. Huckleberry, and J. Wolf, Cycle spaces of flag domains, Progress in Math., vol. 245, Birkhäuser, Boston, MA, USA, 2006.

[GGK13] M. Green, P. Griffiths, and M. Kerr, Hodge theory, complex geometry and representation theory, CBMS Regional Conference Series, vol. 118, AMS, 2013.

[GKa] W. Goldring and J.-S. Koskivirta, Automorphic vector bundles with global sections on G-Zip ${ }^{\mathcal{Z}}$-schemes, Preprint, arXiv:1701.00333.

[GKb] Strata Hasse invariants, Hecke algebras and Galois representations., Preprint, arXiv:1507.05032.

[GS69] P. Griffiths and W. Schmid, Locally homogeneous complex manifolds, Acta. Math. 123 (1969), $253-302$.

[Jan03] J. Jantzen, Representations of algebraic groups, 2nd ed., Math. Surveys and Monographs, vol. 107, American Mathematical Society, Providence, RI, 2003.

[Kis10] M. Kisin, Integral models for Shimura varieties of abelian type, J. Amer. Math. Soc. 23 (2010), no. 4, $967-1012$.

[Kos] J.-S. Koskivirta, Normalization of closed Ekedahl-Oort strata, To appear in Canad. Math. Bull., arXiv:1703.05197.

[KW] J.-S. Koskivirta and T. Wedhorn, Generalized Hasse invariants for Shimura varieties of Hodge type, Preprint, arXiv:1406.2178.

[Mad] K. Madapusi, Toroidal compactifications of integral models of Shimura varieties of Hodge type, Preprint.

[MW04] B. Moonen and T. Wedhorn, Discrete invariants of varieties in positive characteristic, IMRN 72 (2004), $3855-3903$.

[PWZ11] R. Pink, T. Wedhorn, and P. Ziegler, Algebraic zip data, Doc. Math. 16 (2011), 253-300.

[PWZ15] _ F-zips with additional structure, Pacific J. Math. 274 (2015), no. 1, 183-236.

[Vas99] A. Vasiu, Integral canonical models of Shimura varieties of preabelian type, Asian J. Math. 3 (1999), 401-518.

[Wed14] T. Wedhorn, Bruhat strata and F-zips with additional structure, Münster J. Math. 7 (2014), no. 2, 529-556.

[Zha] C. Zhang, Ekedahl-Oort strata for good reductions of Shimura varieties of Hodge type, To appear in Canad. J. Math, arXiv:1312.4869.

(Wushi Goldring) Department of Mathematics, Stockholm University, Stockholm SE-10691, Sweden wushijig@gmail.com

(Jean-Stefan Koskivirta) Department of Mathematics, South Kensington Campus, Imperial College London, London SW7 $2 \mathrm{AZ}, \mathrm{UK}$

jeanstefan.koskivirta@gmail.com 